\title{
IMPROVED CONVERGENCE BOUNDS FOR TWO-LEVEL METHODS WITH AN AGGRESSIVE COARSENING AND MASSIVE POLYNOMIAL SMOOTHING*
}

\author{
RADEK TEZAUR ${ }^{\dagger}$ AND PETR VANĚK ${ }^{\ddagger}$
}

\begin{abstract}
An improved convergence bound for the polynomially accelerated two-level method of Brousek et al. [Electron. Trans. Numer. Anal., 44 (2015), pp. 401-442, Section 5] is proven. This method is a reinterpretation of the smoothed aggregation method with an aggressive coarsening and massive polynomial smoothing of Vaněk, Brezina, and Tezaur [SIAM J. Sci. Comput., 21 (1999), pp. 900-923], and its convergence rate estimate is improved here quantitatively. Next, since the symmetrization of the method requires two solutions of the coarse problem, a modification of the method is proposed that does not have this disadvantage, and a qualitatively better convergence result for the modification is established. In particular, it is shown that a bound of the convergence rate of the method with a multiply ( $k$-times) smoothed prolongator is asymptotically inversely proportional to $d^{2 k}$, where $d$ is the degree of the smoothing polynomial. In earlier works, this acceleration effect is only quadratic. Finally, for another modified multiply smoothed method, it is proved that this convergence improvement is not limited only to an asymptotic regime but holds true everywhere.
\end{abstract}

Key words. two-level method with aggressive coarsening, coarse-space size independent convergence, smoothed aggregation, polynomial smoothing

AMS subject classifications. 65F10, 65N55

1. Introduction. This paper is concerned with an improved convergence analysis of the polynomially accelerated two-level method of [4] and the convergence analysis of its modifications proposed here. The analyzed methods are used for solving linear systems with a positive definite matrix, denoted throughout the paper by $A$. The methods are based on a smoothed aggregation concept where, in order to make small coarse-space sizes possible, we allow for an aggressive coarsening that is compensated for by massive polynomial smoothing. These concepts are briefly reviewed in Section 2 . We show that, for a coarse space characterized by the diameter of the aggregates $H$ and the fine-level space with the mesh size $h$, it is sufficient to use $O(H / h)$ elementary smoothing steps to compensate for the dependence of the convergence rate estimate on the coarsening ratio $H / h$ and get thereby a convergence rate estimate independent of the coarse-space size. The computational cost of our methods is lower than that of the domain decomposition methods that use direct subdomain solvers, as discussed in Section 2 using the bounds of Section 7. This feature is not new here; the methods reviewed in [4] belong to this category. In this paper, we are interested in a radical improvement of the asymptotic convergence bound with respect to the degree of the smoothing polynomial for certain modifications of the methods presented in [4].

An objection can be made that the problem can be solved by a standard multigrid cycle, which would lead to a linear dependence of the overall computational cost on the number of degrees of freedom, with a potentially same level of parallelism, provided that the smoothers are additive. However, when dealing with an algebraic multigrid in particular, it is acceptable to coarsen in a less than optimal fashion once, between the levels one and two, but it is not acceptable to do it systematically and recursively throughout the process of coarsening. This leads either to convergence deterioration and/or to excessive computational costs. Thus, in the context of algebraic multigrid, a two-level frame considered here is more robust.

\footnotetext{
*Received November 1, 2016. Accepted March 12, 2018. Published online on July 2, 2018. Recommended by Marco Donatelli.

${ }^{\dagger}$ Department of Aeronautics and Astronautics, Stanford University, Stanford, CA 94305, USA (rtezaurestanford.edu).

${ }^{\ddagger}$ Department of Mathematics, University of West Bohemia, Univerzitní 22, 30614 Pilsen, Czech Republic (petrvanek09@seznam.cz).
} 
The convergence bound for the method of [4, Section 5] shown therein is a relatively straightforward consequence of a "pointwise" estimate for a general variational two-level framework. It is pointwise in the following sense: the convergence rate estimate is a continuous functional on the Euclidean space of all errors $\mathbb{R}^{n}$; for each error upon an entry of an iteration, it gives a bound of the error reduction factor. To be more precise, assume that $A$ is a symmetric, positive semidefinite matrix, $p: \mathbb{R}^{m} \rightarrow \mathbb{R}^{n}(n=\operatorname{ord}(A), m<n)$ a prolongator that is used by the two-level method, and $E$ the corresponding error propagation operator of a two-level method (for example, $E=S\left(I-p\left(p^{T} A p\right)^{-1} p^{T} A\right.$ ) for a method with the post-smoother $S$ ). The pointwise convergence rate estimate is a continuous functional

$$
r_{\gamma}: \mathbf{e} \in \mathbb{R}^{n} \mapsto \gamma\left(\lambda \frac{\inf _{\mathbf{v}}\|\mathbf{e}-p \mathbf{v}\|^{2}}{|\mathbf{e}|_{A}^{2}}\right) \geq \frac{|E \mathbf{e}|_{A}}{|\mathbf{e}|_{A}},
$$

where $\gamma<1, \gamma(0)=0$, is a continuous increasing function on $\mathbb{R}_{0}^{+}$and $\lambda \geq \varrho(A)$ an available upper bound. Note that $r_{\gamma}$ is invariant with respect to the scaling (multiplication by a scalar factor) of both e and $A$. In Section 3 of this paper, we prove a better pointwise convergence rate estimate for a general variational two-level method with an $A$-symmetric post-smoother and get thereby in Section 4 a sharper estimate for the final polynomially accelerated two-level algorithm. Although only polynomial smoothers are considered here, the pointwise estimate is established for the two-level method with a general $A$-symmetric post-smoother. This general estimate can be used elsewhere. In addition, only the assumption that the smoother is convergent in the $A$-norm is needed here whereas in [4], it is assumed that the error propagation operator of the smoother is positive semidefinite in the $A$-inner product. To summarize, the convergence result for the method of [4] is improved relatively significantly but only quantitatively.

The $A$-symmetrization of the above two-level method, which is necessary for its use as a conjugate gradient method preconditioner, requires two solutions of the coarse-level problem per iteration. In Section 5, we first propose a modification whose $A$-symmetrization does not have this disadvantage, i.e., it requires only one coarse-problem solution per iteration, and we prove a convergence bound for it by different means. This modification uses a double-smoothed prolongator as opposed to a single-smoothed prolongator of the method from [4]. Then, building on the concept of a multiply smoothed prolongator, we consider a method with the multiply smoothed prolongator $P=S^{k} p$ and an adequate multigrid smoother. We prove that if the approximation constant (which reflects approximation properties of the prolongator compared to the "strength" of the smoother $S$ ) is sufficiently small, then the convergence rate estimate is directly proportional to its $k$-th power. This is a useful result since the approximation constant can be made arbitrarily small by using $S$ of a sufficient high degree. In the final convergence theorem, the approximation constant is inversely proportional to the square of the degree of the smoothing polynomial. Thus, the final convergence rate bound is inversely proportional to $d^{2 k}$, where $d$ is the degree of the smoothing polynomial. Then, asymptotically, the method that uses a polynomial of double degree $2 d$ yields an improvement of a factor of $2^{2 k}$ in the convergence rate compared to the method that uses a polynomial of degree $d$. This is achieved at a cost of twice the computational work on the fine-level and under suitable conditions on the construction of the prolongator, four times more computational work in the Cholesky factorization, and twice more work in the backward substitution of the coarse space. We stress that we consider a coarse space of a negligible size, for example, with the dimension equal to the square root of the number of the fine-level degrees of freedom or even fewer on a massively parallel architecture. This justifies the use of higher-degree prolongator smoothers in this method to a greater extent than in our earlier methods since their convergence rate estimate $[3,4,8,10]$ is inversely proportional only to $d^{2}$. 
The radical acceleration of the above method occurs asymptotically only as the approximation constant $C_{A}$ in (4.4) approaches 0 . As stated above, this constitutes only a small weakness as the approximation constant can be made arbitrarily small by using a polynomial smoother of a sufficient high degree. However, in Section 6, we use a more radical (and more expensive) prolongator smoother to achieve the strongest acceleration effect even for $C_{A} \approx 1$.

In Section 7, we apply our framework to the case of a prolongator given by generalized aggregation ([9]) with large aggregates, resulting in a small coarse-space problem. In other words, the aggressive coarsening based on generalized aggregations is balanced by massive smoothing, and the resulting method is optimal in the following sense: for a second-order elliptic problem discretized on a mesh with characteristic mesh size $h$ and a small coarse space characterized by a diameter of the aggregates $H$, the method exhibits a coarse-space size-independent rate of convergence for the cost of $O(H / h)$ elementary smoothing steps. For reasonable aggregates, the coarse-level matrix is sparse. The computational cost of the presented method is asymptotically superior to that of domain decomposition methods that use direct subdomain solvers. In addition, the presented method allows for a finer grain parallelism since the Richardson iterations used as a massive smoother (the bottleneck of the algorithm) therein can be performed using up to $n=\operatorname{ord}(A)$ processors; thus, it can be performed in constant time on an ideal massively parallel architecture. On a real-world massively parallel platform, such as GPU processors, such a smoothing procedure can be performed rapidly. For details, see [4].

Finally, numerical results on a model problem are presented in Section 8. They confirm the theoretical estimates of the previous sections. Conclusions are offered in Section 9.

2. Two-level variational multigrid based on smoothed aggregations. In this section, we define a two-level variational multigrid with a prolongator based on smoothed aggregations, give a brief introduction to the aggregation-based coarsening, and define what we understand as an optimal two-level method with an aggressive coarsening and a massive polynomial smoothing. This introductory section closely follows [4]. The verification that the methods presented in this paper are indeed optimal is presented in Section 7.

The solution of a system of linear algebraic equations

$$
A \mathbf{x}=\mathbf{f}
$$

where $A$ is a symmetric positive definite $n \times n$ matrix that arises from a finite element discretization of an elliptic boundary value problem, is considered. To define a variational twolevel method, two algorithmic ingredients are required: a linear prolongator, $P: \mathbb{R}^{m} \rightarrow \mathbb{R}^{n}$, $m<n$, and a smoothing procedure. Polynomial smoothers that can be expressed as a sequence of Richardson iterations

$$
\mathbf{x} \leftarrow(I-\omega A) \mathbf{x}+\omega \mathbf{f}
$$

are considered here. A particular case of interest is that of a small coarse space, that is, $m \ll n$. Let $\nu_{1}$ and $\nu_{2}$ be integers. A variational two-level method proceeds as follows:

\section{AlgORITHM 1.}

1. For $i=1, \ldots, \nu_{1}$ do $\mathbf{x} \leftarrow\left(I-\alpha_{i} A\right) \mathbf{x}+\alpha_{i} \mathbf{f}$.

2. Set $\mathbf{d}=A \mathbf{x}-\mathbf{f}$.

3. Restrict $\mathbf{d}_{2}=P^{T} \mathbf{d}$.

4. Solve the coarse problem $A_{2} \mathbf{v}=\mathbf{d}_{2}$, where $A_{2}=P^{T} A P$,

5. Correct $\mathrm{x} \leftarrow \mathrm{x}-P \mathbf{v}$,

6. For $i=1, \ldots, \nu_{2}$ do $\mathbf{x} \leftarrow\left(I-\beta_{i} A\right) \mathbf{x}+\beta_{i} \mathbf{f}$. 
In this paper, a tentative prolongator $p$ is constructed by a generalized unknowns aggregation method [9]. A simple example of a (non-generalized) aggregation method is presented below in Example 2.1. For a standard finite element discretization of a scalar elliptic problem, the generalized aggregation method coincides (up to a scaling) with a standard unknowns aggregation [9]. The resulting prolongator $p$ is an orthogonal matrix. The final prolongator $P$ is obtained by polynomial smoothing $[6,7,9,10,11]$

$$
P=S p, \quad S=\left(I-\omega_{1} A\right) \cdots\left(I-\omega_{\nu} A\right),
$$

where $\nu$ is a positive integer. The coefficients $\alpha_{i}, \beta_{i}$, and $\omega_{i}$ are chosen carefully and kept in a close relationship. The tentative prolongator $p$ is responsible for the approximation properties of the coarse space Range $(P)$. The prolongator smoother $S$ enforces smoothness of the coarse-space functions.

EXAMPLE 2.1. Consider a one-dimensional Laplace equation discretized on a uniform mesh that consists of $n=m N$ nodes. A simple unknowns aggregation prolongator can be constructed as follows. Let the nodes be numbered in the usual way from left to right. The aggregates are formed as disjoint sets of $N$ consecutive nodes, i.e.,

$$
\begin{gathered}
\left\{\mathcal{A}_{i}\right\}_{i=1}^{m}=\{\{1,2, \ldots, N\},\{N+1, N+2, \ldots, 2 N\}, \ldots, \\
\{(m-1) N+1, \ldots, m N-1, m N\}\} .
\end{gathered}
$$

The corresponding prolongator is given by

$$
p_{i j}= \begin{cases}1 & \text { iff } i \in \mathcal{A}_{j}, \\ 0 & \text { otherwise }\end{cases}
$$

that is, the $j$-th column is created by restricting a vector of ones onto the $j$-th aggregate with zeroes elsewhere. In matrix form,

$$
\left.p=\left[\begin{array}{cccc}
1 & & & \\
\vdots & & & \\
1 & & & \\
& 1 & & \\
& \vdots & & \\
& 1 & & \\
& & \vdots & \\
& & \vdots & \\
& & \\
& & & \\
& & 1 \\
& & & \\
\mathcal{A}_{2} \\
& & & \\
& & & 1
\end{array}\right]\right\} \mathcal{A}_{m}
$$

The action of the prolongator corresponds to a disaggregation of the $j$-th $\mathbb{R}^{m}$-variable into $N \mathbb{R}^{n}$-variables forming the aggregate $\mathcal{A}_{j}$. Thus, $p$ can be thought of as a discrete piecewise constant interpolation. The prolongator becomes an orthogonal matrix by the scaling

$$
p \leftarrow \frac{1}{\sqrt{N}} p
$$


For scalar problems (such as Example 2.1), the columns of the prolongator $p$ have a disjoint nonzero structure. This can also be viewed as the discrete basis functions of the coarse space Range $(p)$ having disjoint supports. For non-scalar elliptic problems, several fine-level vectors are restricted to each of the aggregates. For example, for a discretization of the equations of linear elasticity in three dimensions, six rigid-body modes are restricted to each of the aggregates, giving rise to six columns with the same nonzero structure. Such a set of columns is labeled a super-column and the corresponding set of coarse-level degrees of freedom (each associated with one column) a super-node. The super-columns have a disjoint nonzero structure corresponding to the disjoint nonzero structure of the aggregates. Thus, in general, it is assumed that the discrete coarse-space basis functions (columns of the prolongator $p$ ) are non-overlapping unless they belong to the same aggregate.

A key assumption to prove convergence of a two-level method is that the prolongator satisfies the weak approximation condition

$$
\forall \mathbf{e} \in \mathbb{R}^{n} \exists \mathbf{v} \in \mathbb{R}^{m}: \quad\|\mathbf{e}-p \mathbf{v}\|^{2} \leq \frac{C_{A}}{\varrho(A)}\left(\frac{H}{h}\right)^{2}\|\mathbf{e}\|_{A}^{2} .
$$

Here, $h$ is a characteristic element size of the fine-level discretization (assuming the quasiuniformity of the mesh), and $H$ is a characteristic diameter of the aggregates (understood as a set of finite element nodal points). A simple example of a verification of (2.2) in one dimension is shown in [4, Section 2] using the Poincaré inequality. For a scalar elliptic second-order problem, (2.2) was proved in [9]. For the case of linear elasticity in 3D, the reader is referred to [10].

The constant in the weak approximation condition (2.2) depends on the ratio $\frac{H}{h}$. As a result, the convergence of a straightforward two-level method depends on the same ratio. More specifically, assuming (2.2), the variational two-level method with the prolongator $p$ and a single Jacobi post-smoothing step converges with the rate of convergence

$$
\|E \mathbf{e}\|_{A}^{2} \leq\left(1-C\left(\frac{h}{H}\right)^{2}\right)\|\mathbf{e}\|_{A}^{2},
$$

where $E$ is the error propagation operator of the method. Our objective is to eliminate the dependence of the convergence of a two-level method on the ratio $\frac{H}{h}$ for a minimal possible cost. Domain decomposition methods strive toward the same goal. A typical domain decomposition method can be viewed as a two-level multigrid method with a small coarse space whose local resolution corresponds to the subdomain size and a block-smoother that uses direct subdomain solvers. The subdomain solvers are relatively expensive. Here, methods with a much lower cost that also open the room for a better level of fine-grain parallelism are described and analyzed.

A two-level method is labeled optimal if, for a second-order elliptic problem discretized on a mesh with the mesh size $h$, it yields a small, sparse coarse space characterized by the diameter $H$ and an $H / h$-independent rate of convergence for the cost of $O(H / h)$ elementary smoothing steps. Generally, a Richardson iteration sweep given by (2.1) is considered as an elementary smoothing step. One cannot possibly expect a better result, i.e., coarse-space size-independent convergence with fewer than $O(H / h)$ smoothing steps, since that many steps are needed to establish essential communication within the discrete distance $O(H / h)$, that is, the continuous distance $O(H)$.

In three dimensions, an optimal two-level method is significantly less expensive than a domain decomposition (DD) method based on direct subdomain solvers. A DD method needs to solve $O\left((1 / H)^{3}\right)$ subdomain linear problems of size $O\left((H / h)^{3}\right)$. When a direct sparse 


\section{ETNA}

Kent State University and

Johann Radon Institute (RICAM)

solver is used for the Cholesky decomposition of the local matrices in the DD method, the total fine-level cost is at least $O\left(1 / H^{3}\left((H / h)^{3}\right)^{2}\right)=O\left(1 / h^{3}(H / h)^{3}\right)=O\left(n(H / h)^{3}\right.$ operations. However, the cost of the optimal two-level method is only $O(n(H / h)$. Furthermore, aside from the cost of communication, an optimal two-level method is more amenable to massive parallelism. The smoothing using $O(H / h)$ Richardson sweeps (2.1), which constitutes a bottleneck of the entire procedure, can be performed using up to $n$ processors. On the other hand, in a DD method, a subdomain-level parallelism, and therefore a much coarser-grain parallelism, is natural, where typically only $O(m)$ processors can be utilized with $m$ being the number of subdomains.

3. Pointwise estimate for a variational two-level method. For the linear system with a symmetric, positive semidefinite $n \times n$ matrix $A$, the variational two-level method with prolongator $p(n \times m$ full-rank matrix, $m<n)$ and common smoothers is known to converge uniformly under the condition:

$$
\exists C>0:\left(\forall \mathbf{e} \in \mathbb{R}^{n} \exists \mathbf{v} \in \mathbb{R}^{m}:\|\mathbf{e}-p \mathbf{v}\|^{2} \leq \frac{C}{\lambda}|\mathbf{e}|_{A}^{2}\right),
$$

where $\lambda \geq \varrho(A)$ is the upper bound used in the smoother. (If the smoother contains no upper bound of $\varrho(A)$, then we can consider $\lambda=\varrho(A)$.) The above assumption is usually called the weak approximation condition and is restated here without the spatial size parameters found in its version (2.2).

The (very simple) theory of [2] then gives the estimate for the $A$-seminorm of the error propagation operator $E$ in the form $|E|_{A} \leq q(C)<1$, that is,

$$
\forall \mathbf{e} \in \mathbb{R}^{n}: \quad|E \mathbf{e}|_{A} \leq q(C)|\mathbf{e}|_{A},
$$

with $q<1$ being a continuous increasing function on $\mathbb{R}^{+}$. In other words, from the uniform validity of the weak approximation condition in (3.1), the uniform error estimate (3.2) follows. By the term "uniform" we understand "valid with the same constant for all e".

In [4], when studying the uniformity of the convergence of a two-level method with an aggressive coarsening and a massive polynomial smoothing, we needed a stronger convergence property of the variational two-level method that is also required in the abstract convergence theory of Section 4 below. The error estimate needs to hold point by point in the sense that it gives an upper bound of the error contraction factor for each particular error and the convergence rate estimate is a continuous functional on the space of all errors $\mathbb{R}^{n}$. The definition of the pointwise convergence estimate, which is in accordance with its sketch in the introduction, follows. In order to be able to treat more general smoothers, we find it useful to formulate the definition in a general Hilbert space setting. For reasons that will become clear in Section 4, we need to allow the semidefiniteness of $A$.

DEFINITION 3.1 (Pointwise convergence estimate). Let $H=\left(\mathbb{R}^{n},\langle\cdot, \cdot\rangle\right)$ be a vector Hilbert space with the induced norm $\|\cdot\|, A$ a self-adjoint positive semidefinite operator on $H$, $|\cdot|_{A}$ the A-seminorm on $H$, and $\lambda \geq \lambda_{\max }(A)$ an upper bound used in the smoother. Assume that there is a continuous increasing function $\gamma(\cdot)<1, \gamma(0)=0$, defined on $\mathbb{R}_{0}^{+}$such that (for the two-level method with the error propagation operator $E$ ) the following implication holds:

$$
\begin{aligned}
& \forall \mathbf{e} \in \mathbb{R}^{n}: \\
& \qquad\left(\exists \mathbf{v} \in \mathbb{R}^{m}, C=C(\mathbf{e}) \geq 0:\|\mathbf{e}-p \mathbf{v}\|^{2} \leq \frac{C}{\lambda}|\mathbf{e}|_{A}^{2} \Longrightarrow|E \mathbf{e}|_{A} \leq \gamma(C)|\mathbf{e}|_{A}\right) .
\end{aligned}
$$

Then we call the estimate (3.3) pointwise. 
For the sake of brevity we simply identify the abstract estimate (3.3) with the continuous functional $r_{\gamma}$ in (1.1) and call the functional $r_{\gamma}$ a pointwise convergence rate estimate.

The pointwise convergence estimate is not easy to establish. In [4], we provided a proof of such a property that is an adaptation of the proof of the multi-level theory of [1]. The proof of [1] cannot be used directly since it requires the stability $|Q|_{A} \leq C$ of the interpolation operator $Q: \mathbf{e} \mapsto p \mathbf{v}$ in (3.3). Possible objections to our adaptation of this proof are:

1. Aesthetic: to use the very complex multi-level proof of [1] is an overkill. Our adaptation of the proof is several pages long and is also extremely technical. This is, of course, not an objection against the masterpiece of [1] itself; in the context of the multi-level method such complexity is adequate and a simpler proof is hardly possible. This is true even about the more recent proof based on the so-called XZ-identity [12].

2. The estimate is not very sharp.

3. The proof is restricted to the case of a smoother with a positive semidefinite error propagation operator (in the $A$-inner semi-product).

In this paper, we provide a much simpler and more straightforward proof that is not restricted to the case of a smoother with a positive semidefinite error propagation operator and that gives an estimate that is, particularly for small $C$, much sharper. For realistic values of $C$, the new estimate is nearly the square of the old estimate of [4]. The strong improvement of the convergence estimate can always be achieved in the resulting polynomially accelerated method since, by using a polynomial of a sufficient high degree, the pointwise estimate is employed with a constant $C$ in the region of the strong acceleration (cf. (4.7) and (5.8))

In [4], we proved an estimate for the variational two-level method that is pointwise according to a different definition, which is equivalent to Definition 3.1 as shown below. The alternative definition reflects how we used the pointwise convergence estimate in the proof of the convergence theorem. The same definition is also needed in Section 4 of this paper. Again, we formulate it in the general Hilbert space setting.

DEFINITION 3.2. Let $\left(\mathbb{R}^{n},\langle\cdot, \cdot\rangle\right)$ be a vector Hilbert space with the induced norm $\|\cdot\|, A$ a self-adjoint positive semidefinite operator on $H,|\cdot|_{A}$ the A-seminorm on $H$, $\lambda \geq \lambda_{\max }(A)$ the upper bound used in the smoother, and $E$ the error propagation operator of the two-level method with the prolongator $p$. Assume that there is a continuous increasing function $\gamma(\cdot)<1, \gamma(0)=0$, defined on $\mathbb{R}_{0}^{+}$such that the following holds:

Assume that, for all $V \subset \mathbb{R}^{n}, V \neq \emptyset$, the uniform validity of the weak approximation condition for all $\mathbf{e} \in V$ with the same constant $C$, i.e.,

$$
\exists C=C(V) \geq 0: \quad\left(\forall \mathbf{e} \in V \exists \mathbf{v} \in \mathbb{R}^{m}:\|\mathbf{e}-p \mathbf{v}\|^{2} \leq \frac{C}{\varrho(A)}|\mathbf{e}|_{A}^{2}\right)
$$

implies the uniform bound

$$
\forall \mathbf{e} \in V: \quad|E \mathbf{e}|_{A} \leq \gamma(C)|\mathbf{e}|_{A} .
$$

Then we call the abstract estimate (3.4) $\Longrightarrow$ (3.5) pointwise and the functional $r_{\gamma}$ in (1.1) a pointwise rate of convergence.

Definitions 3.1 and 3.2 are equivalent, and $\gamma$ is in both cases the same function. Indeed, assume that the convergence estimate that conforms to Definition 3.2 holds true. Let e $\in \mathbb{R}^{n}$. The predicate (3.4) $\Longrightarrow$ (3.5) for $V=\{\mathbf{e}\}$ implies the predicate (3.3) with $C_{(3.3)}(\mathbf{e})=C_{(3.4)}(\{\mathbf{e}\})$. Thus the estimate conforms to Definition 3.1. Assume that the convergence estimate conforms to Definition 3.1. Let $V \subset \mathbb{R}^{n}$. The predicate $\forall \mathbf{e} \in V:$ (3.3) implies the predicate (3.4) $\Longrightarrow(3.5)$ with $C_{(3.4)}(V)=\sup _{\mathbf{e} \in V}\left\{C_{(3.3)}(\mathbf{e})\right\}$. Thus, the convergence estimate conforms to Definition 3.2. The weak approximation condition (3.1) follows from (3.4) with $C_{(3.1)}=C_{(3.4)}\left(\mathbb{R}^{n}\right)$. 
The error propagation operator of a two-level variational method without a pre-smoother is given by $E=S(I-Q)$, where $Q=p\left(p^{T} A p\right)^{+} p^{T} A$ is the $A$-orthogonal projection onto Range $(p)$ ([2]), the symbol ${ }^{+}$denotes the Moore-Penrose pseudoinverse, and $S$ is the error propagation operator of the post-smoother. The following lemma was proved as Lemma 5.4 of [4] based on a regularity-free multi-level estimate of [1].

Lemma 3.3. Let $\left(\mathbb{R}^{n},\langle\cdot, \cdot\rangle\right)$ be the Euclidean space with the induced norm $\|\cdot\|$, A a symmetric positive semidefinite matrix, $\lambda \geq \varrho(A)$, and $p$ an $n \times m$ full-rank matrix, $m<n$. Furthermore, let $R$ be a symmetric positive definite $n \times n$ matrix such that $S \equiv I-R^{-1} A$ is positive semidefinite in the A-inner semi-product. We assume that there is a constant $C_{R}>0$ such that for all $\mathbf{w} \in \mathbb{R}^{n}$

$$
\frac{1}{\lambda}\|\mathbf{w}\|^{2} \leq C_{R}\left\langle R^{-1} \mathbf{w}, \mathbf{w}\right\rangle .
$$

Let $V \subset \mathbb{R}^{n}$. Assume (3.4) holds true. Then for $E=S\left[I-p\left(p^{T} A p\right)^{+} p^{T} A\right]$, the inequality (3.5) is satisfied with

$$
\gamma(C)=\left(1-\frac{1}{1+C C_{R}}\right)^{1 / 2}
$$

As an alternative to Lemma 3.3, we provide the following statement with a much simpler proof. This statement is not limited to the case of a smoother with a positive semidefinite error propagation operator. The only assumption here is that the choice of the parameters ensures that the smoother is convergent in the $A$-seminorm. The estimate is also sharper than that of Lemma 3.3.

THEOREM 3.4. Let $H=\left(\mathbb{R}^{n},\langle\cdot, \cdot\rangle\right)$ be a vector Hilbert space with the induced norm $\|\cdot\|$, A a symmetric positive semidefinite operator on $H,|\cdot|_{A}$ the A-seminorm on $H$,

$$
p:\left(\mathbb{R}^{m},\langle\cdot, \cdot\rangle_{2}: \mathbf{x}, \mathbf{y} \mapsto \sum_{i=1}^{m} x_{i} y_{i}\right) \rightarrow H
$$

a linear injective mapping, and $p^{*}$ the adjoint operator. Let $Q=p\left(p^{*} A p\right)^{+} p^{*} A$ and $S=I-\omega / \lambda A$, where $\lambda \geq \lambda_{\max }(A)$ is an available upper bound, and $\omega \in(0,2)$. For $E=S(I-Q)$, the implication (3.3) is satisfied with

$$
\gamma(C)= \begin{cases}\left(\frac{C}{4 \omega(2-\omega)}\right)^{1 / 2} & \text { for } C \leq 2 \omega(2-\omega) \\ \left(1-\frac{\omega(2-\omega)}{C}\right)^{1 / 2} & \text { for } C>2 \omega(2-\omega)\end{cases}
$$

Proof. Let $\mathbf{e} \in \mathbb{R}^{n}, \mathbf{e}_{1}=(I-Q) \mathbf{e}$, and let the left-hand side of the implication (3.3) be satisfied. If $\mathbf{e}_{1} \in \operatorname{Ker}(A)$, then $S \mathbf{e}_{1}=\mathbf{e}_{1} \in \operatorname{Ker}(A)$, and the right-hand side of the implication (3.3) holds true trivially. Thus, assume $\mathbf{e}_{1} \notin \operatorname{Ker}(A)$, which implies $\mathbf{e} \notin \operatorname{Ker}(A)$, and define $t=\left|\mathbf{e}_{1}\right|_{A} /|\mathbf{e}|_{A}$. Since the operator $I-Q$ is an $A$-orthogonal projection, $t \in(0,1]$. Thus, the left-hand side of the implication (3.3) holds, and the fact that $\operatorname{Range}(Q) \subset \operatorname{Range}(p)$ yields

$$
\left\|\mathbf{e}_{1}-p \mathbf{v}_{1}\right\|^{2} \leq C / \lambda|\mathbf{e}|_{A}^{2} \leq \frac{C}{t^{2} \lambda}\left|\mathbf{e}_{1}\right|_{A}^{2},
$$

where $\mathbf{v}_{1}=\mathbf{v}-\left(p^{*} A p\right)^{+} p^{*} A \mathbf{e}$. 
TABLE 3.1

Comparison of the new convergence rate estimate $\gamma$ (3.7) with the old estimate $\gamma_{\text {old }}$ (3.6) for the Richardson smoother $I-\omega / \lambda$ A and $\omega=1$.

\begin{tabular}{c|c|c|c}
$C$ & $\gamma(C)$ & $\gamma_{\text {old }}(C)$ & $\alpha: \gamma_{\text {old }}^{\alpha}=\gamma$ \\
\hline 0.1 & 0.158 & 0.301 & 1.536 \\
0.5 & 0.353 & 0.577 & 1.893 \\
1.0 & 0.500 & 0.707 & 1.999 \\
2.0 & 0.707 & 0.816 & 1.705 \\
5.0 & 0.894 & 0.912 & 1.216
\end{tabular}

Next, we use a classical argument by Achi Brandt [2] based on the orthogonality argument known from the proof of Céa's lemma ([5]):

$$
\begin{aligned}
\left|S \mathbf{e}_{1}\right|_{A}^{2} & =\left|\mathbf{e}_{1}\right|_{A}^{2}-2 \frac{\omega}{\lambda}\left\|A \mathbf{e}_{1}\right\|^{2}+\left(\frac{\omega}{\lambda}\right)^{2}\left|A \mathbf{e}_{1}\right|_{A}^{2} \leq\left|\mathbf{e}_{1}\right|_{A}^{2}-2 \frac{\omega}{\lambda}\left\|A \mathbf{e}_{1}\right\|^{2}+\frac{\omega^{2}}{\lambda}\left\|A \mathbf{e}_{1}\right\|^{2} \\
& =\left|\mathbf{e}_{1}\right|_{A}^{2}\left(1-\frac{\omega(2-\omega)}{\lambda} \frac{\left\|A \mathbf{e}_{1}\right\|^{2}}{\left|\mathbf{e}_{1}\right|_{A}^{2}}\right) .
\end{aligned}
$$

Since $Q$ is the $A$-orthogonal projection onto $\operatorname{Range}(p), I-Q$ is the $A$-orthogonal projection onto Range $(p)^{\perp_{A}} \equiv\left\{\mathbf{v} \in \mathbb{R}^{n}:\langle A \mathbf{v}, p \cdot\rangle=0\right\}$, and we have $\left\langle A \mathbf{e}_{1}, p \mathbf{v}_{1}\right\rangle=0$. Using this property, (3.8), and the Cauchy-Schwarz inequality, we get the estimate

$$
\left|\mathbf{e}_{1}\right|_{A}^{2}=\left\langle A \mathbf{e}_{1}, \mathbf{e}_{1}-p \mathbf{v}_{1}\right\rangle \leq\left\|A \mathbf{e}_{1}\right\|\left\|\mathbf{e}_{1}-p \mathbf{v}_{1}\right\| \leq \sqrt{C /\left(t^{2} \lambda\right)}\left\|A \mathbf{e}_{1}\right\|\left|\mathbf{e}_{1}\right|_{A} .
$$

Dividing the above inequality by $\left|\mathbf{e}_{1}\right|_{A}$ and squaring the result gives the coercivity bound

$$
\frac{\left\|A \mathbf{e}_{1}\right\|^{2}}{\left|\mathbf{e}_{1}\right|_{A}^{2}} \geq \frac{t^{2} \lambda}{C} .
$$

Substituting this estimate into (3.9) and using $t \in(0,1]$ yields

$$
\frac{|S(I-Q) \mathbf{e}|_{A}^{2}}{|\mathbf{e}|_{A}^{2}}=\frac{\left|S \mathbf{e}_{1}\right|_{A}^{2}}{\left|\mathbf{e}_{1}\right|_{A}^{2}} \frac{\left|\mathbf{e}_{1}\right|_{A}^{2}}{|\mathbf{e}|_{A}^{2}} \leq t^{2}\left(1-\frac{t^{2} \omega(2-\omega)}{C}\right) \leq \max _{\xi \in[0,1]} \xi\left(1-\frac{\xi \omega(2-\omega)}{C}\right) .
$$

The right-hand side of the above inequality is a maximum of a concave quadratic function in $\xi$ on the interval $[0,1]$. The global maximum is attained for $\hat{\xi}=C /[2 \omega(2-\omega)] \geq 0$. Assuming $C /[2 \omega(2-\omega)] \in[0,1]$, the global maximum is also the maximum on the interval $[0,1]$ and equals $C /[4 \omega(2-\omega)]$. Let $C /[2 \omega(2-\omega)]>1$. The maximized concave quadratic function is increasing on $(-\infty, C /[2 \omega(2-\omega)]] \supset[0,1]$, hence increasing on $[0,1]$. The maximum on $[0,1]$ is therefore attained for $\xi=1$ and equals to $1-\omega(2-\omega) / C$. This proves the right-hand side of the implication (3.3) with the function $\gamma$ given by (3.7). The fact that the function $\gamma$ conforms to Definition 3.1 is now evident.

REMARK 3.5. The proof of Theorem 3.4 provides a natural insight into the functioning of the method when the constant $C$ in (3.3) is small. By (3.10), $C / t \geq 1$, hence $t=|(I-Q) \mathbf{e}|_{A}^{2} /|\mathbf{e}|_{A}^{2} \leq C$, that is, the coarse-level correction must be efficient for $C<1$.

Theorem 3.4 with the choice $H \equiv \mathbb{R}^{n}$ provides the pointwise convergence estimate for the Richardson smoother $I-\omega / \lambda A$. An improvement of the new estimate (3.7) compared to the old estimate (3.6) is demonstrated in Table 3.1, where the convergence rate $\gamma_{\text {old }}$ is given by a formula $\gamma_{o l d}=\sqrt{1-1 /(1+C / \omega)}, \omega \in(0,1]$. The value $\omega=1$ is therefore optimal with respect to both $\gamma$ and $\gamma_{\text {old }}$.

In the rest of this section we prove an estimate for a smoother with the error propagation operator $I-\omega / \lambda_{R} R^{-1} A$, where $R$ is a symmetric, positive definite matrix, $\lambda_{R}$ is an upper 
bound of $\lambda_{\max }\left(R^{-1} A\right)$, and $\omega \in(0,2)$. The parameter $\omega$ is chosen so that the smoother is convergent in the $A$-seminorm. If $R$ is nonsymmetric, then it can be symmetrized by performing an iteration with $R$ followed by an iteration with $R^{T}$. The estimate follows from Theorem 3.4 using the substitutions

$$
H \leftarrow\left(\mathbb{R}^{n},\langle\cdot, \cdot\rangle_{R}\right), \quad A \leftarrow R^{-1} A,
$$

where $\langle\cdot, \cdot\rangle_{R}$ and $\|\cdot\|_{R}$ are the usual $R$-inner product and the induced $R$-norm, respectively. We prove the following corollary:

COROLlaRY 3.6. Let $A$ be a symmetric, positive semidefinite $n \times n$ matrix, $R$ a symmetric, positive definite $n \times n$ matrix, and $p$ an $n \times m$ full-rank matrix, $m<n$. Let $Q=p\left(p^{T} A p\right)^{+} p^{T} A$ and $S_{R}=I-\omega / \lambda_{R} R^{-1} A$, where $\lambda_{R} \geq \lambda_{\max }\left(R^{-1} A\right)$ is an available upper bound, and $\omega \in(0,2)$. Then, $E=S_{R}(I-Q)$ satisfies the pointwise convergence estimate (3.3) with $\gamma$ given by (3.7) and $\|\cdot\| \leftarrow\|\cdot\|_{R}$.

Proof. Let $H$ and $A$ in Theorem 3.4 be given by (3.11). Clearly, $R^{-1} A$ is an $\langle\cdot, \cdot\rangle_{R^{-}}$ symmetric, positive semidefinite operator. Then $\lambda_{\max }(A)$ of Theorem 3.4 becomes $\lambda_{\max }\left(R^{-1} A\right), \lambda$ of Theorem 3.4 becomes $\lambda_{R}$, the smoother $S$ of Theorem 3.4 becomes $I-\omega / \lambda_{R} R^{-1} A=S_{R}$, and $p^{*}$ of Theorem 3.4 becomes $p^{T} R$. Hence $Q$ of Theorem 3.4 becomes

$$
p\left(p^{T} R\left(R^{-1} A\right) p\right)^{+} p^{T} R\left(R^{-1} A\right)=p\left(p^{T} A p\right)^{+} p^{T} A .
$$

Since the inner product of Theorem 3.4 becomes $\left\langle R^{-1} A \cdot, \cdot\right\rangle_{R}=\langle A \cdot, \cdot\rangle$, the seminorm of Theorem 3.4 becomes $|\cdot|_{A}$. The statement now follows by Theorem 3.4.

4. An improved convergence bound for the method of [4, Section 5]. In this section, we prove an improved abstract convergence estimate for the polynomially accelerated twolevel method of [4, Section 5], i.e., the two-level method with the error propagation operator

$$
E=S\left(I-\frac{\omega}{\lambda_{A_{S}}} A_{S}\right)\left[I-p\left(p^{T} A_{S} p\right)^{+} p^{T} A_{S}\right], \quad A_{S}=S^{2} A, \quad k \geq 0, \omega \in(0,2),
$$

where $\lambda_{A_{S}} \geq \varrho\left(A_{S}\right)$ is an available upper bound and $S$ a polynomial in $A$ of the form

$$
S=\left(I-\alpha_{1} A\right)\left(I-\alpha_{2} A\right) \cdots\left(I-\alpha_{d} A\right), \quad d \geq 1,
$$

that satisfies $\varrho(S) \leq 1$. Clearly, the corresponding two-level method consists of two parts: the inner iteration with the error propagation operator

$$
E_{A_{S}}=\left(I-\frac{\omega}{\lambda_{A_{S}}} S^{2} A\right)\left[I-p\left(p^{T} A_{S} p\right)^{+} p^{T} A_{S}\right]
$$

and the outer post-smoothing iteration with the error propagation operator $S$. The inner iteration $E_{A_{S}}$ corresponds to the variational multigrid with prolongator $p$ used for the transformed problem with the matrix $A_{S}=S^{2} A$. The outer iteration $S$ is a multiple Richardson smoothing procedure.

It is computationally more efficient to implement the method as a smoothed prolongator method ([10]) with the prolongator $P=S p$ and the identical error propagation operator

$$
\left(I-\frac{\omega}{\lambda_{A_{S}}} A_{S}\right)\left[I-P\left(P^{T} A P\right)^{+} P^{T} A\right] S=E,
$$

where the pre-smoother is interpreted as a sequence of Richardson iterations. 
Following the methodology of [4], we prove the following theorem. The proof follows as a more or less straightforward consequence of Corollary 3.6.

THEOREM 4.1. Let A be a symmetric, positive definite $n \times n$ matrix, $p$ a full-rank $n \times m$ matrix, $m<n$. Further let $S$ be a polynomial in $A$ in the form (4.2) such that $\varrho(S) \leq 1, E$ and $A_{S}$ be given by (4.1), and $\lambda_{A_{S}} \geq \varrho\left(A_{S}\right)$ be an available upper bound. Assume that

$$
\exists C_{A}>0: \quad\left(\forall \mathbf{e} \in \mathbb{R}^{n} \exists \mathbf{v} \in \mathbb{R}^{m}:\|\mathbf{e}-p \mathbf{v}\|^{2} \leq \frac{C_{A}}{\lambda_{A_{S}}}\|\mathbf{e}\|_{A}^{2}\right) .
$$

Then $\|E\|_{A} \leq \gamma\left(C_{A}\right)$ with $\gamma$ given by (3.7).

Proof. Let $\mathbf{e} \in \mathbb{R}^{n}$. We prove $\|E \mathbf{e}\|_{A} \leq \gamma\left(C_{A}\right)\|\mathbf{e}\|_{A}$. We can restrict ourselves to the non-trivial case $\mathbf{e} \notin \operatorname{Ker}(S)$. For every $\tau \in(0,1]$, define the set

$$
V(\tau)=\left\{\mathbf{v} \in \mathbb{R}^{n}:|\mathbf{v}|_{A_{S}} \geq \tau\|\mathbf{v}\|_{A}\right\} \backslash\{\mathbf{0}\} .
$$

Then the assumption (4.4) yields

$$
\forall \mathbf{u} \in V(\tau) \exists \mathbf{v} \in \mathbb{R}^{m}: \quad\|\mathbf{u}-p \mathbf{v}\|^{2} \leq \frac{C_{A} / \tau^{2}}{\lambda_{A_{S}}}|\mathbf{u}|_{A_{S}}^{2} .
$$

Let $t=|\mathbf{e}|_{A_{S}} /\|\mathbf{e}\|_{A}$. Since $\varrho(S) \leq 1, t \in(0,1]$. Trivially, $\mathbf{e} \in V(t)$. Let $E_{A_{S}}$ be given by (4.3). From (4.5) and $\mathbf{e} \in V(t)$, by Corollary 3.6 with $R^{-1} \leftarrow A$ and $A \leftarrow A_{S}$, it follows that $\left|E_{A_{S}} \mathbf{e}\right|_{A_{S}} \leq \gamma\left(C_{A} / t^{2}\right)|\mathbf{e}|_{A_{S}}$. By this estimate, $t \in(0,1]$, and $E=S E_{A_{S}}$, we get

$$
\frac{\|E \mathbf{e}\|_{A}}{\|\mathbf{e}\|_{A}}=\frac{\left|E_{A_{S}} \mathbf{e}\right|_{A_{S}}}{|\mathbf{e}|_{A_{S}}} \frac{|\mathbf{e}|_{A_{S}}}{\|\mathbf{e}\|_{A}} \leq t \gamma\left(C_{A} / t^{2}\right) \leq \sup _{\xi \in(0,1]}\left\{\xi \gamma\left(C_{A} / \xi^{2}\right)\right\} .
$$

Similarly to the end of the proof of Theorem 3.4, inspecting (the limits at) the ends of the interval and the local maxima reveals that the maximum is attained for $\xi=1$, which proves our statement.

REMARK 4.2. In view of Theorem 4.1, a smoothing polynomial $S$ is sought such that it is an error propagation operator of an $A$-non-divergent smoother and makes $\varrho\left(A_{S}\right)=\varrho\left(S^{2} A\right)$ as small as possible. The smaller the available upper bound $\lambda_{A_{S}}$ of $\varrho\left(A_{S}\right)$ is, the easier it becomes to satisfy (4.4) with a small constant $C_{A}$. Such optimal property is held by the linearly transformed Chebyshev polynomial in $A$ given by

$$
q(A)=\left(1-\frac{1}{r_{1}} A\right) \cdots\left(1-\frac{1}{r_{d}} A\right), \quad r_{i}=\frac{\lambda}{2}\left(1-\cos \frac{2 i \pi}{2 d+1}\right),
$$

where $\lambda$ is an available upper bound of $\varrho(A)$. It follows from [4], Lemma 4.4 that for $S=q(A)$,

$$
\lambda_{A_{S}}=\frac{\lambda}{[1+2 \operatorname{deg}(q)]^{2}} \geq \varrho\left(S^{2} A\right)
$$

and that the smoother $S=q(A)$ satisfies $\varrho(S) \leq 1$. Assumption (4.4) then becomes

$$
\exists C_{A}>0: \quad\left(\forall \mathbf{e} \in \mathbb{R}^{n} \exists \mathbf{v} \in \mathbb{R}^{m}:\|\mathbf{e}-p \mathbf{v}\|^{2} \leq \frac{C_{A}[1+2 \operatorname{deg}(S)]^{2}}{\lambda}\|\mathbf{e}\|_{A}^{2}\right)
$$

and is, for larger $d=\operatorname{deg}(S)$, much easier to satisfy with a uniform constant than (3.1). Note that the case of interest is when (3.1) is satisfied with a non-uniform constant $C \equiv c_{0}(H / h)^{2}$ with $c_{0}>0$ independent of $H$ and $h$, where $h$ is the fine and $H$ the coarse-space resolution and the smoother $S$ of a degree $c H / h, c>0$. This yields (4.8) with $C_{A}$ independent of $H / h$. For details, see Section 7. 
5. The $A$-symmetric modifications and their convergence analysis. The $A$-symmetrization of the method in (4.1) requires two solutions of the coarse-level problem per iteration. We investigate a modified method that does not have this disadvantage. Its error propagation operators $E$ and the error propagation operator of its $A$-symmetrization $E_{s}=E E^{*}$ are given by

$$
E=S S_{A_{S}}\left(I-Q_{A}\right), \quad E_{s}=S S_{A_{S}}\left(I-Q_{A}\right) S_{A_{S}} S,
$$

respectively, where

$$
\begin{aligned}
P & =S^{2} p \\
Q_{A} & =P\left(P^{T} A P\right)^{+} P^{T} A, \quad A_{S}=S^{2} A, \quad S_{A_{S}}=I-\frac{\omega}{\lambda_{A_{S}}} A_{S},
\end{aligned}
$$

with $\omega \in(0,2)$, and where $\lambda_{A_{S}}$ is an available upper bound of $\varrho\left(A_{S}\right)$. Thus, we investigate a method with the double-smoothed prolongator $P=S^{2} p$.

Note that, if the coarse-level matrix for the single-smoothed prolongator $(S p)^{T} A(S p)=$ $p^{T} A S^{2} p$ is sparse, then the coarse-level matrix for the double-smoothed prolongator $p^{T} A S^{4} p$ is also sparse and has only several times more non-zero entries and only about twice larger bandwidth (assuming a suitable numbering of the degrees of freedom). The same holds true for the prolongator $P$. In any case, it is an empirical observation of the authors that enlarging the supports of the coarse-space basis functions (by additional prolongator smoothing) until a very fast convergence is achieved pays off in terms of overall performance. This observation is also supported theoretically as elaborated in Remark 5.3 below. Practically, a very small coarse space can be used. For example, for $3 D$ problems, setting the number of coarse degrees of freedom equal to nearly the square root of the number of degrees of freedom on the fine-level is optimal on a serial computational architecture. On a massively parallel platform the cost of the fine-level computations scales linearly until the number of processors $n$ is reached, making an even smaller coarse-space size optimal.

THEOREM 5.1. Let A be a symmetric, positive definite $n \times n$ matrix, $p$ a full-rank $n \times m$ matrix, $m<n$. Furthermore, let $S$ be a polynomial in A of the form (4.2) such that $\varrho(S) \leq 1$. Under assumption (4.4), the error propagation operator $E$ and the error propagation operator of the A-symmetrization $E_{s}=E E^{*}$ given by (5.1) with (5.2) and (5.3) satisfy the estimates $\|E\|_{A} \leq \gamma\left(C_{A}\right)$ and $\left\|E_{s}\right\|_{A} \leq \gamma^{2}\left(C_{A}\right)$, respectively, where $\gamma$ is given by (3.7).

Proof. Let $\mathbf{e} \in \mathbb{R}^{n}$ and $\mathbf{e}_{1}=\left(I-Q_{A}\right) \mathbf{e}$. We assume $\mathbf{e}, \mathbf{e}_{1} \notin \operatorname{Ker}(S)$ since $E \mathbf{e}=\mathbf{0}$ otherwise. Let $t=\left\|\mathbf{e}_{1}\right\|_{A_{S}} /\|\mathbf{e}\|_{A}$. Since $\varrho(S) \leq 1, I-Q_{A}$ is an $A$-orthogonal projection, and $\mathbf{e}, \mathbf{e}_{1} \notin \operatorname{Ker}(S), t \in(0,1]$. By using (3.9) with $A \leftarrow A_{S}$ and $S \leftarrow S_{A_{S}}$, we obtain

$$
\|E \mathbf{e}\|_{A}=\left\|S S_{A_{S}} \mathbf{e}_{1}\right\|_{A}^{2}=\left|S_{A_{S}} \mathbf{e}_{1}\right|_{A_{S}}^{2} \leq t^{2}\left(1-\frac{\omega(2-\omega)}{\lambda_{A_{S}}} \frac{\left\|A_{S} \mathbf{e}_{1}\right\|^{2}}{\left|\mathbf{e}_{1}\right|_{A_{S}}^{2}}\right)\|\mathbf{e}\|_{A}^{2} .
$$

Let $\mathbf{v}=\operatorname{argmin}_{\mathbf{x} \in \mathbb{R}^{m}}\left\|\mathbf{e}_{1}-p \mathbf{x}\right\|$. Since $I-Q_{A}$ is the $A$-orthogonal projection onto $\operatorname{Range}\left(S^{2} p\right)^{\perp_{A}},\left\langle A_{S} \mathbf{e}_{1}, p \mathbf{v}\right\rangle=0$. From this and (4.4) we get

$$
\begin{aligned}
\left|\mathbf{e}_{1}\right|_{A_{S}}^{2} & =\left\langle A_{S} \mathbf{e}_{1}, \mathbf{e}_{1}-p \mathbf{v}\right\rangle \leq\left\|A_{S} \mathbf{e}_{1}\right\|\left\|\mathbf{e}_{1}-p \mathbf{v}\right\| \leq \sqrt{C_{A} / \lambda_{A_{S}}}\left\|A_{S} \mathbf{e}_{1}\right\|\left\|\mathbf{e}_{1}\right\|_{A} \\
& =\sqrt{C_{A} /\left(t^{2} \lambda_{A_{S}}\right)}\left\|A_{S} \mathbf{e}_{1}\right\|\left|\mathbf{e}_{1}\right|_{A_{S}} .
\end{aligned}
$$

Dividing the above estimate by $\left|\mathbf{e}_{1}\right|_{A_{S}}$ and squaring the result yields

$$
\frac{\left\|A_{S} \mathbf{e}_{1}\right\|^{2}}{\left|\mathbf{e}_{1}\right|_{A_{S}}^{2}} \geq t^{2} \frac{\lambda_{A_{S}}}{C_{A}} .
$$


Substituting this estimate into (5.4), we obtain

$$
\frac{\|E \mathbf{e}\|_{A}^{2}}{\|\mathbf{e}\|_{A}^{2}} \leq t^{2}\left(1-\frac{\omega(2-\omega)}{C_{A}} t^{2}\right) \leq \max _{\xi \in[0,1]}\left\{\xi\left(1-\frac{\omega(2-\omega)}{C_{A}} \xi\right)\right\}=\gamma^{2}\left(C_{A}\right),
$$

where the last step has been verified at the end of the proof of Theorem 3.4. This gives $\|E\|_{A} \leq \gamma\left(C_{A}\right)$. The statement $\left\|E_{s}\right\|_{A} \leq \gamma^{2}\left(C_{A}\right)$ follows by

$$
\left\|E_{s}\right\|_{A}=\left\|E E^{*}\right\|_{A} \leq\|E\|_{A}\left\|E^{*}\right\|_{A}=\|E\|_{A}^{2} .
$$

The use of the prolongator $P=S^{2} p$ as in (5.1) is very powerful and theoretically interesting. To demonstrate it, we investigate a modification of the method of (5.1) with the prolongator $P=S^{k} p, k \geq 2$, and $k$ multigrid post-smoothing steps $S^{k}$ instead of a single one $S$. The convergence estimate exhibits a great improvement for $C_{A}<\omega(2-\omega) /(k-1)$, where its dependence on $C_{A}$ is asymptotically of the $k$-th power.

THEOREM 5.2. Let $A$ be a symmetric, positive definite $n \times n$ matrix, $p$ a full-rank $n \times m$ matrix, $m<n$. Furthermore, let $S$ be a polynomial in $A$ of the form (4.2) such that $\varrho(S) \leq 1$, $P=S^{k} p, k \geq 2$, and $Q_{A}, A_{S}$, and $S_{A_{S}}$ are defined as in (5.3). Under assumption (4.4), the error propagation operators $E=S_{A_{S}} S^{k}\left(I-Q_{A}\right)$ and $E_{s}=E E^{*}=S_{A_{S}} S^{k}\left(I-Q_{A}\right) S^{k} S_{A_{S}}$ satisfy $\|E\|_{A} \leq \gamma_{2}\left(C_{A}\right)$ and $\left\|E_{s}\right\|_{A} \leq \gamma_{2}^{2}\left(C_{A}\right)$ with the function $\gamma_{2}$ given by

$$
\gamma_{2}^{2}\left(C_{A}\right)= \begin{cases}\frac{C_{A}^{k}}{\left[C_{A}+\omega(2-\omega)\right]^{k}} & \text { for } C_{A} \in\left[0, \frac{\omega(2-\omega)}{k-1}\right), \\ \frac{1}{k}\left[\frac{k-1}{\omega(2-\omega) k}\right]^{k-1} C_{A}^{k-1} & \text { for } C_{A} \in\left[\frac{\omega(2-\omega)}{k-1}, \omega(2-\omega) \frac{k}{k-1}\right), \\ 1-\frac{\omega(2-\omega)}{C_{A}} & \text { for } C_{A} \in\left[\omega(2-\omega) \frac{k}{k-1}, \infty\right) .\end{cases}
$$

Proof. Let $\mathbf{e} \in \mathbb{R}^{n}$ and $\mathbf{e}_{1}=\left(I-Q_{A}\right)$ e. We can assume that $\mathbf{e}, \mathbf{e}_{1} \notin \operatorname{Ker}(S)$. Let $\mathbf{e}_{2}=S^{k-2} \mathbf{e}_{1}$. Since $S$ and $A_{S}$ commute, $\|S\|_{A} \leq 1$ and $\left\|S_{A_{S}}\right\|_{A} \leq 1$, we have $\left\|S_{A_{S}} S^{2} \mathbf{e}_{2}\right\|_{A} \leq\left\|S^{2} \mathbf{e}_{2}\right\|_{A}$ and $\left\|S_{A_{S}} S^{2} \mathbf{e}_{2}\right\|_{A}=\left\|S S_{A_{S}} S \mathbf{e}_{2}\right\|_{A} \leq\left\|S_{A_{S}} S \mathbf{e}_{2}\right\|_{A}$. From here it follows that

$$
\frac{\left\|S_{A_{S}} S^{2} \mathbf{e}_{2}\right\|_{A}}{\left\|\mathbf{e}_{2}\right\|_{A}} \leq \min \left\{\frac{\left\|S^{2} \mathbf{e}_{2}\right\|_{A}}{\left\|\mathbf{e}_{2}\right\|_{A}}, \frac{\left\|S_{A_{S}} S \mathbf{e}_{2}\right\|_{A}}{\left\|\mathbf{e}_{2}\right\|_{A}}\right\} .
$$

Let $\mathbf{v}=\operatorname{argmin}_{\mathbf{x} \in \mathbb{R}^{m}}\left\|S^{2} \mathbf{e}_{2}-p \mathbf{v}\right\|$. Since $I-Q_{A}$ is the $A$-orthogonal projection onto Range $\left(S^{k} p\right)^{\perp_{A}}, \mathbf{e}_{2} \in \operatorname{Range}\left(S^{2} p\right)^{\perp_{A}}=\operatorname{Range}(p)^{\perp_{A_{S}}}$. We estimate using this orthogonality, the Cauchy-Schwarz inequality, assumption (4.4), and the identity $\left\|S^{2} \mathbf{e}_{2}\right\|_{A}=\left|S \mathbf{e}_{2}\right|_{A_{S}}$ as follows:

$$
\left|S \mathbf{e}_{2}\right|_{A_{S}}^{2}=\left\langle A_{S} \mathbf{e}_{2}, S^{2} \mathbf{e}_{2}-p \mathbf{v}\right\rangle \leq\left\|A_{S} \mathbf{e}_{2}\right\|\left\|S^{2} \mathbf{e}_{2}-p \mathbf{v}\right\| \leq \sqrt{C_{A} / \lambda_{A_{S}}}\left|S \mathbf{e}_{2}\right|_{A_{S}}\left\|A_{S} \mathbf{e}_{2}\right\| .
$$

Dividing the estimate by $\left|S \mathbf{e}_{2}\right|_{A_{S}}$ and squaring the result yields

$$
\frac{\left\|A_{S} \mathbf{e}_{2}\right\|^{2}}{\left|S \mathbf{e}_{2}\right|_{A_{S}}^{2}} \geq \frac{\lambda_{A_{S}}}{C_{A}} .
$$

Let $t_{j}=\left\|S^{j} \mathbf{e}_{1}\right\|_{A} /\left\|S^{j-1} \mathbf{e}_{1}\right\|_{A}, j=1, \ldots, k$. From the definition of $\mathbf{e}_{2}$, it follows that $t_{k-1}=\left|\mathbf{e}_{2}\right|_{A_{S}} /\left\|\mathbf{e}_{2}\right\|_{A}$ and $t_{k}=\left|S \mathbf{e}_{2}\right|_{A_{S}} /\left\|S \mathbf{e}_{2}\right\|_{A}=\left|S \mathbf{e}_{2}\right|_{A_{S}} /\left|\mathbf{e}_{2}\right|_{A_{S}}$. Clearly,

$$
\left|\mathbf{e}_{2}\right|_{A_{S}}^{2}=\left\langle A S^{2} \mathbf{e}_{2}, \mathbf{e}_{2}\right\rangle \leq\left\|S^{2} \mathbf{e}_{2}\right\|_{A}\left\|\mathbf{e}_{2}\right\|_{A}=\left|S \mathbf{e}_{2}\right|_{A_{S}}\left\|\mathbf{e}_{2}\right\|_{A}
$$




\section{ETNA}

Kent State University and

Johann Radon Institute (RICAM)

Dividing the estimate by $\left|\mathbf{e}_{2}\right|_{A_{S}}\left\|\mathbf{e}_{2}\right\|_{A}$ yields $t_{k-1} \leq t_{k}$. Thus, $0<t_{k-1} \leq t_{k} \leq 1$. From (5.4) and (5.7) it follows that

$$
\frac{\left\|S S_{A_{S}} \mathbf{e}_{2}\right\|_{A}^{2}}{\left\|\mathbf{e}_{2}\right\|_{A}^{2}}=t_{k-1}^{2}\left(1-t_{k}^{2} \frac{\omega(2-\omega)}{\lambda_{A_{S}}} \frac{\left\|A_{S} \mathbf{e}_{2}\right\|^{2}}{\left|S \mathbf{e}_{2}\right|_{A_{S}}^{2}}\right) \leq t_{k-1}^{2}\left(1-t_{2}^{2} \frac{\omega(2-\omega)}{C_{A}}\right) .
$$

By the above estimate, the second inequality of (5.6), and $\left\|S^{2} \mathbf{e}_{2}\right\|_{A}=t_{k-1} t_{k}\left\|\mathbf{e}_{2}\right\|_{A}$, we get

$$
\frac{\left\|S_{A_{S}} S^{2} \mathbf{e}_{2}\right\|_{A}^{2}}{\left\|\mathbf{e}_{2}\right\|_{A}^{2}} \leq t_{k-1}^{2} \min \left\{t_{k}^{2}, 1-t_{k}^{2} \frac{\omega(2-\omega)}{C_{A}}\right\}, \quad 0<t_{k-1} \leq t_{k} \leq 1 .
$$

Hence, since $t_{k}$ are non-decreasing,

$$
\begin{aligned}
\frac{\|E \mathbf{e}\|_{A}^{2}}{\|\mathbf{e}\|_{A}^{2}} & =\frac{\left\|S_{A_{S}} S^{2} \mathbf{e}_{2}\right\|_{A}^{2}}{\left\|\mathbf{e}_{2}\right\|_{A}^{2}} \frac{\left\|\mathbf{e}_{2}\right\|_{A}^{2}}{\|\mathbf{e}\|_{A}^{2}} \leq\left(t_{1} \cdots t_{k-1}\right)^{2} \min \left\{t_{k}^{2}, 1-t_{k}^{2} \frac{\omega(2-\omega)}{C_{A}}\right\} \\
& \leq t_{k-1}^{2(k-1)} \min \left\{t_{k}^{2}, 1-t_{k}^{2} \frac{\omega(2-\omega)}{C_{A}}\right\} \leq \max _{\left[\xi_{1}, \xi_{2}\right] \in T} \xi_{1}^{k-1} \min \left\{\xi_{2}, 1-\xi_{2} \frac{\omega(2-\omega)}{C_{A}}\right\},
\end{aligned}
$$

where $T=\left\{\left[\xi_{1}, \xi_{2}\right]: 0 \leq \xi_{1} \leq \xi_{2} \leq 1\right\}$. Since the argument is increasing in $\xi_{1}$, the maximum is attained for $\xi_{1}=\xi_{2}$. Inspecting the maximum of this function of one variable yields the statement $\|E\|_{A} \leq \gamma_{2}\left(C_{A}\right)$, and $\left\|E_{s}\right\|_{A} \leq \gamma_{2}^{2}\left(C_{A}\right)$ follows by the symmetrization argument.

REMARK 5.3. Let the assumption (4.8) for $d=\operatorname{deg}(S)=0$ be satisfied with $C_{A} \equiv C_{A, d=0}$. Then for a polynomial $S=q(A)$ given by (4.6) of degree $d>0$, the assumption (4.8) holds true with

$$
C_{A} \equiv C_{A, d}=C_{A, d=0} /(1+2 d)^{2} .
$$

Note that for second-order elliptic problems discretized on a mesh with the characteristic resolution $h$ and a coarse space with the characteristic resolution $H$, (4.8) holds for $d=0$ with a non-uniform constant $C_{A, d=0}=O\left((H / h)^{2}\right)$. For details, see Section 7 .

The estimate of the rate of convergence of the symmetrized method of Theorem 5.2 is asymptotically proportional to $C_{A, d}^{k}$ for a small $C_{A, d}$. This combined with (5.8) implies that the estimate is inversely proportional to $(1+2 d)^{2 k}$. In our previous convergence results $[3,4,8,10]$, the convergence rate bound is inversely proportional to $(1+2 d)^{2}$ only. It is a relevant result since by using $S$ as the polynomial $q(A)$ in (4.6) of a sufficient degree $d$, the constant $C_{A, d}$ can be made arbitrarily small and kept within the region with the strong acceleration. Thus, considering the polynomial $S$ of degree $2 d$ instead of $d$ in the asymptotic region should improve the convergence rate almost $2^{2 k}$ times and incurs only twice more computational work on the fine level.

Taking into account the factor two in the computational work (and assuming that the convergence rate estimate is sharp and the cost of the coarse-level correction is negligible; see below), let us now compare an error reduction effect of one iteration that uses the smoother of degree $2 d$ with the effect of two iterations that use the smoother of degree $d$. Assuming the optimal choice $\omega=1$ and $d$ already large enough so that $(1+2(2 d))^{2} /(1+2 d)^{2} \approx 4$, cf. (5.8), the corresponding error reduction is given by $\gamma_{2}^{2}\left(C_{A, d} / 4\right)$ (approximately) and $\gamma_{2}^{4}\left(C_{A, d}\right)$. Assuming $k=2$ or $k=3$ and inspecting where $\gamma_{2}^{4}\left(C_{A, d}\right)=\gamma_{2}^{2}\left(C_{A, d} / 4\right)$, we find that this holds true for $C_{A, d}=1 / 2$, and the corresponding error reduction factor is then $1 / 3^{2}$ or $1 / 3^{3}$, respectively. Thus, for $C_{A, d}>1 / 2$, it pays off to enlarge twice the degree of the smoothing polynomial rather than perform two iterations with the smoother of degree $d$. For $C_{A, d}<1 / 2$, the opposite is true. If $C_{A, d}=1 / 2$, then the method that uses the 
smoother of degree $2 d$ and two iterations of the method that uses a polynomial of degree $d$ are comparably efficient, and increasing twice the degree of $S$ still does not represent a waste of computational resources. The optimal $d$ is therefore when $C_{A, d} \in[1 / 8,1 / 2]$, which corresponds to convergence rates in the interval $\left[1 / 9^{k}, 1 / 3^{k}\right]$. A similar conclusion can be drawn for $k>3$.

The calculation above presumes that the cost of the coarse-level correction is negligible, i.e., the coarse-space problem is sufficiently small. Otherwise, we would also need to account for the fact that, when we increase the degree $d=O(H / h)$ twice, the coarse-level problem bandwidth increases about twice (assuming a suitable numbering of degrees of freedom) making the setup phase of the Cholesky decomposition on the coarse-level about 4 times more expensive and the forward-backward coarse solutions about twice expensive.

REMARK 5.4. A consequence of our theory is an observation that, at least asymptotically, it is much better to use a prolongator $P=S^{k} p, k>1$, than a single Chebyshev prolongator smoother of degree $k d$.

6. More radical prolongator smoothing. In this section, we prove a convergence bound for a modification of the method of Theorem 5.2. An estimate superior to the sharpest bound of Theorem 5.2 valid only for small $C_{A}$ there is valid here for any $C_{A} \approx 1$. For a larger $C_{A}$, the new convergence rate estimate is the $k$-th power of the old one. The computational complexity of the method presented here is larger, but the strong acceleration effect is achieved under a much weaker condition.

The modified method employs the prolongator $P=\left(S_{A_{S}} S\right)^{k} S^{2} p, k \geq 0$, and the postsmoother $\left(S_{A_{S}} S\right)^{k+1}$. Below, we investigate its convergence and the convergence of its symmetrization.

THEOREM 6.1. Let A be a symmetric, positive definite $n \times n$ matrix, $p$ a full-rank $n \times m$ matrix, $m<n$, and $S$ a polynomial in $A$ of the form (4.2) such that $\varrho(S) \leq 1$. Denote $A_{S}=S^{2} A$ and $S_{A_{S}}=I-\omega / \lambda_{A_{S}} A_{S}$, where $\omega \in(0,2)$ and $\lambda_{A_{S}}$ is an available upper bound of the spectral radius $\varrho\left(A_{S}\right)$. Let the prolongator be given as $P=\left(S_{A_{S}} S\right)^{k} S^{2} p, k \geq 0$, and denote $Q=P\left(P^{T} A P\right)^{-1} P^{T} A$.

Then, under assumption (4.4), the error propagation operator $E=\left(S_{A_{S}} S\right)^{k+1}(I-Q)$ of a two-level variational method characterized by the prolongator $P$ and a smoother with the error propagation operator $\left(S_{A_{S}} S\right)^{k+1}$ and the error propagation operator $E_{s}=E E^{*}=$ $\left(S_{A_{S}} S\right)^{k+1}(I-Q)\left(S_{A_{S}} S\right)^{k+1}$ of its A-symmetrization (where* denotes the A-adjoint operator) satisfy $\|E\|_{A} \leq \gamma^{k+1}\left(C_{A}\right)$ and $\left\|E_{s}\right\|_{A} \leq \gamma^{2(k+1)}\left(C_{A}\right)$ with $\gamma$ given by (3.7).

Proof. Let $\mathbf{e} \in \mathbb{R}^{n}, S^{\prime}=S_{A_{S}} S, \mathbf{e}_{1}=(I-Q) \mathbf{e}$, and $\mathbf{e}_{2}=\left(S^{\prime}\right)^{k} \mathbf{e}_{1}$. We prove first $\|E \mathbf{e}\|_{A} \leq \gamma^{k+1}\left(C_{A}\right)\|\mathbf{e}\|_{A}$. If $\mathbf{e} \in \operatorname{Ker}(S), \mathbf{e}_{1} \in \operatorname{Ker}(S)$ or $\mathbf{e}_{2} \in \operatorname{Ker}(S)$, then $E \mathbf{e}=\mathbf{0}$ and the desired inequality holds trivially. Thus, we can assume that $\mathbf{e}, \mathbf{e}_{1}, \mathbf{e}_{2} \notin \operatorname{Ker}(S)$.

First, we will show that

$$
\frac{\|E \mathbf{e}\|_{A}}{\|\mathbf{e}\|_{A}} \leq\left(\frac{\left\|S^{\prime} \mathbf{e}_{2}\right\|_{A}}{\left\|\mathbf{e}_{2}\right\|_{A}}\right)^{k+1}
$$

Indeed, since $I-Q$ is an $A$-orthogonal projection, $\left\|\mathbf{e}_{1}\right\|_{A} \leq\|\mathbf{e}\|_{A}$, and therefore

$$
\frac{\|E \mathbf{e}\|_{A}}{\|\mathbf{e}\|_{A}} \leq \frac{\left\|\left(S^{\prime}\right)^{k+1} \mathbf{e}_{1}\right\|_{A}}{\left\|\mathbf{e}_{1}\right\|_{A}}=\frac{\left\|\left(S^{\prime}\right)^{k+1} \mathbf{e}_{1}\right\|_{A}}{\left\|\left(S^{\prime}\right)^{k} \mathbf{e}_{1}\right\|_{A}} \frac{\left\|\left(S^{\prime}\right)^{k} \mathbf{e}_{1}\right\|_{A}}{\left\|\left(S^{\prime}\right)^{k-1} \mathbf{e}_{1}\right\|_{A}} \cdots \frac{\left\|S^{\prime} \mathbf{e}_{1}\right\|_{A}}{\left\|\mathbf{e}_{1}\right\|_{A}} .
$$

Let $0<i \leq k$. We have

$$
\left\|\left(S^{\prime}\right)^{i} \mathbf{e}_{1}\right\|_{A}^{2}=\left\langle A\left(S^{\prime}\right)^{i+1} \mathbf{e}_{1},\left(S^{\prime}\right)^{i-1} \mathbf{e}_{1}\right\rangle \leq\left\|\left(S^{\prime}\right)^{i+1} \mathbf{e}_{1}\right\|_{A}\left\|\left(S^{\prime}\right)^{i-1} \mathbf{e}_{1}\right\|_{A} .
$$


Dividing this estimate by $\left\|\left(S^{\prime}\right)^{i} \mathbf{e}_{1}\right\|_{A}\left\|\left(S^{\prime}\right)^{i-1} \mathbf{e}_{1}\right\|_{A}$ yields

$$
\frac{\left\|\left(S^{\prime}\right)^{i} \mathbf{e}_{1}\right\|_{A}}{\left\|\left(S^{\prime}\right)^{i-1} \mathbf{e}_{1}\right\|_{A}} \leq \frac{\left\|\left(S^{\prime}\right)^{i+1} \mathbf{e}_{1}\right\|_{A}}{\left\|\left(S^{\prime}\right)^{i} \mathbf{e}_{1}\right\|_{A}} \leq \frac{\left\|\left(S^{\prime}\right)^{k+1} \mathbf{e}_{1}\right\|_{A}}{\left\|\left(S^{\prime}\right)^{k} \mathbf{e}_{1}\right\|_{A}}=\frac{\left\|S^{\prime} \mathbf{e}_{2}\right\|_{A}}{\left\|\mathbf{e}_{2}\right\|_{A}} .
$$

This together with (6.2) proves (6.1).

Since $I-Q$ is the $A$-orthogonal projection onto Range $\left(\left(S^{\prime}\right)^{k} S^{2}\right)^{\perp_{A}}$, where $\perp_{A}$ denotes the $A$-orthogonal complement, we have $\mathbf{e}_{2}=\left(S^{\prime}\right)^{k} \mathbf{e}_{1} \in \operatorname{Range}\left(S^{2} p\right)^{\perp_{A}}$. Next, we prove that

$$
\forall \mathbf{x} \in \operatorname{Range}\left(S^{2} p\right)^{\perp_{A}} \backslash\{\mathbf{0}\}: \quad \frac{\left\|S^{\prime} \mathbf{x}\right\|_{A}^{2}}{\|\mathbf{x}\|_{A}^{2}} \leq \gamma\left(C_{A}\right) .
$$

The proof of (6.3) follows the proof of Theorem 5.1. We have

$$
\begin{aligned}
& \left\|S^{\prime} \mathbf{x}\right\|_{A}^{2}=\left\|S_{A_{S}} \mathbf{x}\right\|_{A_{S}}^{2}=\|\mathbf{x}\|_{A_{S}}^{2}-2 \frac{\omega}{\lambda_{A_{S}}}\|\mathbf{x}\|_{A_{S}}^{2}+\left(\frac{\omega}{\lambda_{A_{S}}}\right)^{2}\left\|A_{S} \mathbf{x}\right\|_{A_{S}}^{2} \\
& \text { (6.4) } \leq\|\mathbf{x}\|_{A_{S}}^{2}-2 \frac{\omega}{\lambda_{A_{S}}}\left\|A_{S} \mathbf{x}\right\|^{2}+\frac{\omega^{2}}{\lambda_{A_{S}}}\left\|A_{S} \mathbf{x}\right\|^{2}=\left(1-\frac{\omega(2-\omega)}{\lambda_{A_{S}}} \frac{\left\|A_{S} \mathbf{x}\right\|^{2}}{\|\mathbf{x}\|_{A_{S}}^{2}}\right)\|\mathbf{x}\|_{A_{S}}^{2} .
\end{aligned}
$$

Set $\mathbf{v}=\operatorname{argmin}_{\mathbf{w}}\|\mathbf{x}-p \mathbf{w}\|$. Since $\mathbf{x} \in \operatorname{Range}\left(S^{2} p\right)^{\perp_{A}}$, we have $\left\langle A_{S} \mathbf{x}, p \cdot\right\rangle=0$. We set $t=\|\mathbf{x}\|_{A_{S}} /\|\mathbf{x}\|_{A}$ and estimate using this orthogonality, assumption (4.4), and the CauchySchwarz inequality:

$$
\begin{aligned}
\|\mathbf{x}\|_{A_{S}}^{2} & =\left\langle A_{S} \mathbf{x}, \mathbf{x}-p \mathbf{v}\right\rangle \leq\left\|A_{S} \mathbf{x}\right\|\|\mathbf{x}-p \mathbf{v}\| \leq \sqrt{C_{A} / \lambda_{A_{S}}}\left\|A_{S} \mathbf{x}\right\|\|\mathbf{x}\|_{A} \\
& \leq \sqrt{C_{A} /\left(t^{2} \lambda_{A_{S}}\right)}\left\|A_{S} \mathbf{x}\right\|\|\mathbf{x}\|_{A_{S}} .
\end{aligned}
$$

Dividing this estimate by $\|\mathbf{x}\|_{A_{S}}$ and squaring the result yields

$$
\frac{\left\|A_{S} \mathbf{x}\right\|^{2}}{\|\mathbf{x}\|_{A_{S}}^{2}} \geq t^{2} \frac{\lambda_{A_{S}}}{C_{A}} .
$$

Clearly, $t \in(0,1]$. Substituting (6.5) into (6.4) yields

$$
\frac{\left\|S^{\prime} \mathbf{x}\right\|_{A}^{2}}{\|\mathbf{x}\|_{A}^{2}} \leq t^{2}\left(1-\frac{\omega(2-\omega)}{C_{A}} t^{2}\right) \leq \max _{\xi \in[0,1]} \xi\left(1-\frac{\omega(2-\omega)}{C_{A}} \xi\right) .
$$

The estimate (6.3) follows by the same calculations as those used at the end of the proof of Theorem 3.4. Since $\mathbf{e}_{2} \in \operatorname{Range}\left(S^{2} p\right)^{\perp_{A}}$ and $\mathbf{e} \neq \mathbf{0} \in \operatorname{Ker}(S)$, (6.3) holds for $\mathbf{x}=\mathbf{e}_{2}$ and $\|E\|_{A} \leq \gamma^{k+1}\left(C_{A}\right)$ follows by (2.3). Finally,

$$
\left\|E_{s}\right\|_{A}=\left\|E E^{*}\right\|_{A} \leq\|E\|_{A}\left\|E^{*}\right\|_{A}=\|E\|_{A}^{2} \leq \gamma^{2(k+1)}\left(C_{A}\right) .
$$

REMARK 6.2. Let us consider $\omega=1$ and a large enough $d=\operatorname{deg}(S)$ so that, using (5.8), $C_{A, d} \approx 1$ and $C_{A, 2 d} \approx \frac{1}{4} C_{A, d}$. Then, the respective estimates of the rate of convergence $\gamma^{2(k+1)}\left(C_{A, d}\right)$ and $\gamma^{2(k+1)}\left(C_{A, 2 d}\right)$ of the symmetrized method with the degree of $S$ equal to $d$ and $2 d$ satisfy

$$
\gamma^{2(k+1)}\left(C_{A, 2 d}\right) \approx \frac{1}{4^{k+1}} \gamma^{2(k+1)}\left(C_{A, d}\right) .
$$

In Section 5, this was true only asymptotically for $C_{A, d} \rightarrow 0$. Here, the assumption $C_{A, d} \approx 1$ is sufficient. 
7. Application to the method with an aggressive coarsening based on the unknowns aggregation and a massive polynomial smoothing. We apply the results of Sections 4, 5, and 6 to a system arising from a finite element discretization of a second-order elliptic PDE. Throughout this section, the prolongator $p$ is assumed to be constructed by a generalized unknowns aggregation method ([9]). A brief introduction to the unknowns aggregation coarsening is included in Section 2.

The resulting methods feature an aggressive coarsening (resulting in a small coarse space) based on the unknowns aggregation, balanced by a massive polynomial smoothing (a multiple Richardson iteration). They are optimal in the following sense: for a secondorder elliptic problem discretized on a mesh with the characteristic mesh size $h$ and a coarse space characterized by the resolution $H$, they exhibit a coarse-space size independent rate of convergence for the cost of $O(H / h)$ elementary smoothing steps. The coarse-level matrix is sparse if the aggregates have a reasonably compact shape and approximately the same size.

The theory of the previous sections can be readily applied, provided that the prolongator satisfies a version of the weak approximation condition required by the method. In order to achieve coarse-space size and problem size independent convergence for the considered methods, it is necessary to establish that the prolongator satisfies

$$
\exists C>0: \quad\left(\forall \mathbf{e} \in \mathbb{R}^{n} \exists \mathbf{v} \in \mathbb{R}^{m}:\|\mathbf{e}-p \mathbf{v}\|^{2} \leq \frac{C}{\lambda}\left(\frac{H}{h}\right)^{2}\|\mathbf{e}\|_{A}^{2}\right)
$$

with a constant $C$ independent of $h$ and $H$. Here, $h$ is a characteristic element size of the fine-level discretization (assuming the quasi-uniformity of the mesh), $H$ is a characteristic diameter of the aggregates (understood as a set of finite element nodal points), and $\lambda$ is an available upper bound of $\varrho(A)$. For a scalar elliptic second-order problem, (7.1) was proved in [9]. For the case of linear elasticity in 3D, the reader is referred to [10].

We summarize the results in the following uniform theorem:

THEOREM 7.1. Let A be a symmetric, positive definite $n \times n$ matrix, $p$ a full-rank $n \times m$ matrix, $m<n$. Assume the prolongator p satisfies (7.1), the smoother $S$ is given by (4.6), its degree $d$ satisfies $d \geq c_{d} H / h$ with $c_{d}>0$, and $\lambda_{A_{S}}$ is given by (4.7). Then the error propagation operator $E$ in both (4.1) and (5.1) and $E_{s}$ in (5.1) satisfy

$$
\|E\|_{A} \leq \gamma\left(\frac{C_{(7.1)}}{4 c_{d}^{2}}\right), \quad\left\|E_{s}\right\|_{A} \leq \gamma^{2}\left(\frac{C_{(7.1)}}{4 c_{d}^{2}}\right),
$$

where $C_{(7.1)}$ is the constant $C$ in (7.1) and the function $\gamma$ is given by (3.7). The operators $E$ and $E_{s}$ of Theorem 5.2 satisfy

$$
\|E\|_{A} \leq \gamma_{2}\left(\frac{C_{(7.1)}}{4 c_{d}^{2}}\right), \quad\left\|E_{s}\right\|_{A} \leq \gamma_{2}^{2}\left(\frac{C_{(7.1)}}{4 c_{d}^{2}}\right),
$$

with the function $\gamma_{2}$ given by (5.5). Furthermore, for the method of Section 6, we have

$$
\|E\|_{A} \leq \gamma^{k+1}\left(\frac{C_{(7.1)}}{4 c_{d}^{2}}\right), \quad\left\|E_{s}\right\|_{A} \leq \gamma^{2(k+1)}\left(\frac{C_{(7.1)}}{4 c_{d}^{2}}\right),
$$

with $\gamma$ given by (3.7). In (7.2), (7.3), and (7.4), the rate of convergence is independent of $h$ and $H$.

Proof. For $\lambda_{A_{S}}$ in (4.7) we have

$$
\lambda_{A_{S}} \leq \frac{\lambda}{\left(1+2 c_{d} H / h\right)^{2}} \leq \frac{\lambda}{4 c_{d}^{2}}\left(\frac{h}{H}\right)^{2},
$$




\section{ETNA}

Kent State University and

Johann Radon Institute (RICAM)

which together with (7.1) yields that (4.4) is satisfied with

$$
C_{A} \leq C_{(7.1)}\left(\frac{H}{h}\right)^{2} \frac{\lambda_{A_{S}}}{\lambda} \leq \frac{C_{(7.1)}}{4 c_{d}^{2}} .
$$

Then, the estimates (7.2), (7.3), and (7.4) follow from Theorems 4.1, 5.1, 5.2, and 6.1, respectively.

REMARK 7.2. In view of Theorem 7.1, we choose $d=\operatorname{deg}(S)$ equal to $c(H / h)$. A reasonable choice is $c=1 / 2$. For the $A$-symmetric method of Theorem 5.2, based on Remark 5.3, we strive to choose $c$ so that the final convergence rate is about 0.01-0.1.

8. Numerical experiments. The purpose of this section is to illustrate the convergence of the proposed methods and verify that the convergence estimates obtained in the previous sections are predictive of the actual numerical convergence. To this end, the methods are implemented in MATLAB and the focus here is on the number of iterations and the convergence rate. Numerical experiments comparing the efficiency of the presented methods and other domain decomposition methods, preferably on a parallel platform, are beyond the scope of this theoretical paper.

A numerical solution of the Poisson equation is considered in a three-dimensional cube. A Dirichlet boundary condition is imposed on the left, top, and bottom faces. The cube is discretized using bilinear quadrilateral finite elements $\left(Q_{1}\right)$ on two uniform grids of $60 \times 60 \times 60$ and $120 \times 120 \times 120$ elements, resulting in systems of approximately 216 thousand and 1.728 million degrees of freedom, respectively.

The tentative prolongator is constructed from elemental partitions into subdomains of size $10 \times 10 \times 10$ and $20 \times 20 \times 20$ elements. Each of the interior subdomain nodes is assigned to its aggregate (a column of the prolongator) and the interface nodes are assigned to the column with the lower index. The constant $\omega$ is set to 1 throughout. The iterations are started from a zero initial guess, and they are stopped when the relative residual

$$
\frac{\|A \mathbf{x}-\mathbf{f}\|}{\|\mathbf{f}\|}
$$

drops below $10^{-6}$ or the number of iterations exceeds 100 . Table 8.1 displays the number of iterations required by each method for the two discretizations and the two aggregate sizes and a varying degree of the smoothing polynomial $d$. In the table, the methods are denoted as follows:

A6S5O - Algorithm 6 of [4, Section 5];

T5.1 - Algorithm of Theorem 5.1;

T5.1s - Symmetrized algorithm of Theorem 5.1;

T5.2k2 - Algorithm of Theorem 5.2 with $k=2$;

T5.2sk2 - Symmetrized algorithm of Theorem 5.2 with $k=2$;

T5.2k3 - Algorithm of Theorem 5.2 with $k=3$;

T5.2sk3 - Symmetrized algorithm of Theorem 5.2 with $k=3$.

Due to its cost, the method of Section 6 is interesting mostly theoretically and is not considered in the comparisons.

Table 8.1, displays the iteration counts for all of the methods above, the two problem sizes, and the two aggregates sizes when the degree of the smoothing polynomial $d$ is varied. Comparing the rows corresponding to the same $c_{d}$ (cf. Theorem 7.1), e.g., the problem $120 \times 120 \times 120$ for $d=3$ with aggregates of size $10 \times 10 \times 10$ and $d=6$ with aggregates of size $20 \times 20 \times 20$, one can observe that the iteration counts remain virtually the same, confirming the theoretical estimates. Furthermore, the iteration counts remain almost constant 


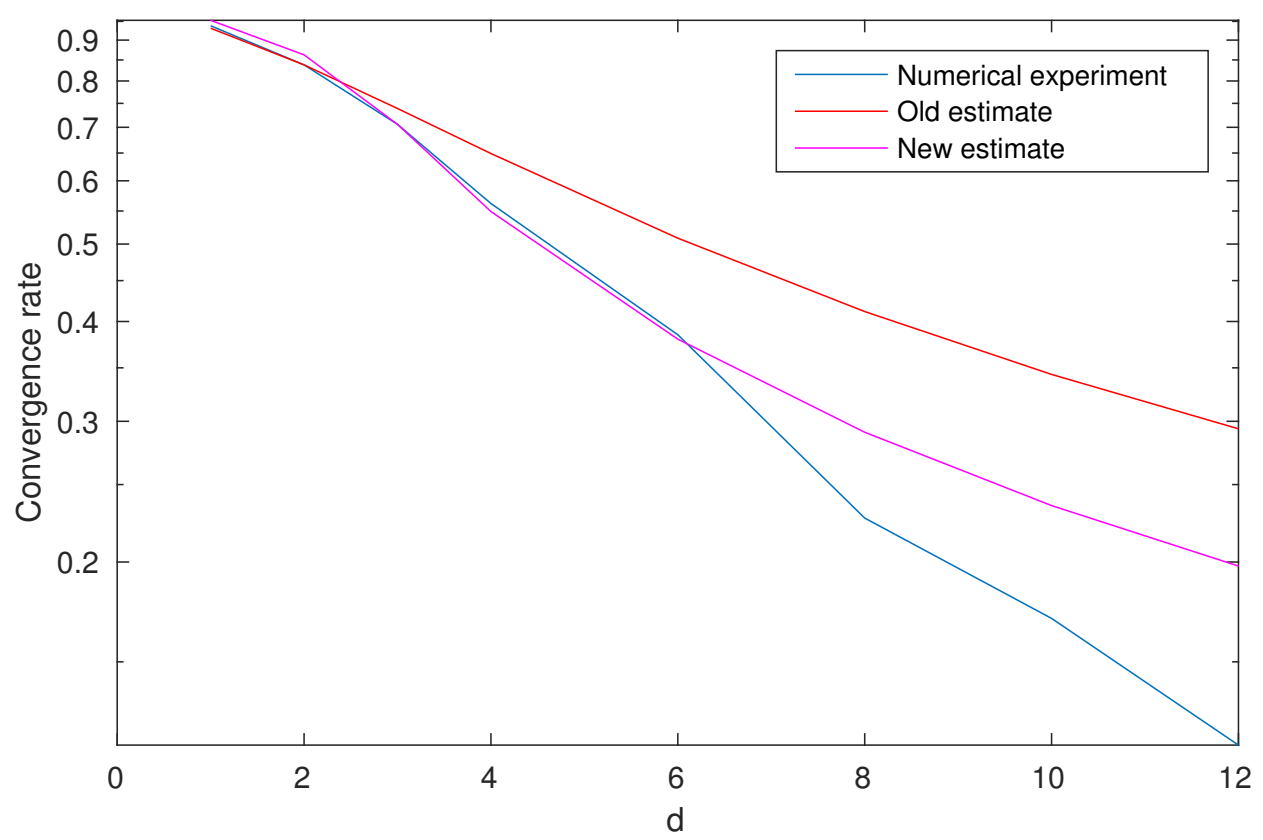

FIG. 8.1. Numerical rates of convergence compared to the old and new theoretical estimates for $120 \times 120 \times 120$ cube with subdomains of size $20 \times 20 \times 20$ and the method of [4, Section 5].

with respect to the problem size when keeping $d$ and $H / h$ constant as can be seen by comparing the entries in the first and third or the second and fourth blocks of the table.

The convergence rate of the methods in the matrix $A$-norm, which is used in the estimates of the theorems, are tabulated in Table 8.2. The rates are computed from the last two iterations of each run. The same conclusions that can be observed from Table 8.1 for the number of iterations can also be drawn for the convergence rates: the convergence rates are virtually independent of the coarse-space size when the degree of polynomial is adjusted to keep $c_{d}$ constant. The validity of comments from Remark 5.3 can also be seen. For example, considering the larger $120 \times 120 \times 120$ problem with subdomains of size $20 \times 20 \times 20$ and the symmetrized method of Theorem 5.2 with $k=2$, the ratio of convergence rates for $d=6$ and $d=2 \cdot 6=12$ is 34 , even exceeding the estimate of approximately $2^{2 k}=16$. Similarly, in this case for the method with $k=3$, the ratio is 409 , again exceeding the estimate of $2^{2 k}=64$.

Finally, the convergence rates from Table 8.2 are used to compare the improved estimate of Section 4 to that of [4, Section 5]. For this purpose, the problem with $120 \times 120 \times 120$ elements and aggregates of size $20 \times 20 \times 20$ is considered, and the convergence rates from the first column are plotted in Figure 8.1 as a function of the degree of the smoothing polynomial $d$. Then, the constants from the new estimates and the old estimate are calibrated to the numerical rate for $d=1$ and $d=2$, respectively, and the rest of their dependence is plotted using (3.7) with (5.8) and the old estimate $\sqrt{1-1 /(1+C)}$ from [4]. It can be seen that the old estimate greatly underestimates the convergence rates. The new estimate matches up the numerical convergence rate up to $d=6$ and is considerably closer than the old one throughout the range of considered values.

9. Conclusions. We have proven that for a two-level variational multigrid method with a multiply ( $k$-times) smoothed prolongator and an adequate multigrid smoother, asymptotically (i.e., for a sufficient large degree of the smoothing polynomial $d=\operatorname{deg}(S)$ ), the convergence 
bound improves greatly with increasing $\mathrm{d}$. Current and future work is focusing on a more practical version of the method of Section 6 whose convergence rate improves greatly even for moderate $d$ and a high-performance implementation of the methods.

\section{REFERENCES}

[1] J. H. BRAmble, J. E. PASciak, J. P. WANG, AND J. Xu, Convergence estimates for multigrid algorithms without regularity assumptions, Math. Comp., 57 (1991), pp. 23-45.

[2] A. BRANDT, Algebraic multigrid theory: the symmetric case, Appl. Math. Comput., 19 (1986), pp. 23-56.

[3] M. BREZINA, P. VANĚK, AND P. S. VASSILEVSKI, An improved convergence analysis of smoothed aggregation algebraic multigrid, Numer. Linear Algebra Appl., 19 (2012), pp. 441-469.

[4] J. Brousek, P. Franková, M. Hanuš, H. Kopincová, R. Kužel, R. Tezaur, P. VaněK, and $\mathrm{Z}$. VASTL, An overview of multilevel methods with aggressive coarsening and massive polynomial smoothing, Electron. Trans. Numer. Anal., 44 (2015), pp. 401-442. http://etna.ricam.oeaw.ac.at/vol.44.2015/pp401-442.dir/pp401-442.pdf

[5] P. G. Ciarlet, The Finite Element Method for Elliptic Problems, North-Holland, Amsterdam, 1978.

[6] P. VANĚK, Acceleration of convergence of a two-level algorithm by smoothing transfer operators, Appl. Math., 37 (1992), pp. 265-274.

[7] — Fast multigrid solver, Appl. Math., 40 (1995), pp. 1-20.

[8] P. VANĚK AND M. BREZINA, Nearly optimal convergence result for multigrid with aggressive coarsening and polynomial smoothing, Appl. Math., 58 (2013), pp. 369-388.

[9] P. VANĚK, M. BREZINA, AND J. MANDEL, Convergence of algebraic multigrid based on smoothed aggregation, Numer. Math., 88 (2001), pp. 559-579.

[10] P. VANĚK, M. BREZINA, AND R. TEZAUR, Two-grid method for linear elasticity on unstructured meshes, SIAM J. Sci. Comput., 21 (1999), pp. 900-923.

[11] P. VANĚK, J. MANDEL, AND M. BREZINA, Algebraic multigrid by smoothed aggregation for second and fourth order elliptic problems, Computing, 56 (1996), pp. 179-196.

[12] J. XU AND L. ZIKATANOV, The method of alternating projections and the method of subspace corrections in Hilbert space, J. Amer. Math. Soc., 15 (2002), pp. 573-597. 
TABLE 8.1

Number of iterations by method and degree of the smoothing polynomial.

\begin{tabular}{|c|c|c|c|c|c|c|c|}
\hline$d$ & A6S5O & T5.1 & $\mathrm{T} 5.1 \mathrm{~s}$ & $\mathrm{~T} 5.2 \mathrm{k} 2$ & T5.2sk2 & $\mathrm{T} 5.2 \mathrm{k} 3$ & $\mathrm{~T} 5.2 \mathrm{sk} 3$ \\
\hline \multicolumn{8}{|c|}{$60 \times 60 \times 60$ with aggregates of size $10 \times 10 \times 10$} \\
\hline 1 & 63 & 56 & 33 & 50 & 31 & 44 & 25 \\
\hline 2 & 23 & 18 & 12 & 16 & 11 & 14 & 9 \\
\hline 3 & 16 & 9 & 6 & 8 & 6 & 6 & 5 \\
\hline 4 & 10 & 7 & 4 & 5 & 4 & 4 & 3 \\
\hline 6 & 7 & 6 & 4 & 4 & 3 & 3 & 2 \\
\hline 8 & 7 & 6 & 3 & 3 & 3 & 2 & 2 \\
\hline 10 & 6 & 5 & 3 & 3 & 2 & 2 & 2 \\
\hline 12 & 6 & 5 & 3 & 3 & 2 & 2 & 2 \\
\hline \multicolumn{8}{|c|}{$60 \times 60 \times 60$ with aggregates of size $20 \times 20 \times 20$} \\
\hline 1 & $100+$ & $100+$ & $100+$ & $100+$ & $100+$ & $100+$ & 89 \\
\hline 2 & 80 & 69 & 40 & 61 & 38 & 53 & 30 \\
\hline 3 & 41 & 33 & 21 & 30 & 20 & 26 & 15 \\
\hline 4 & 26 & 19 & 13 & 17 & 12 & 15 & 9 \\
\hline 6 & 15 & 8 & 6 & 8 & 6 & 6 & 4 \\
\hline 8 & 11 & 7 & 4 & 5 & 3 & 3 & 3 \\
\hline 10 & 8 & 7 & 4 & 4 & 2 & 3 & 2 \\
\hline 12 & 7 & 6 & 4 & 4 & 3 & 3 & 2 \\
\hline
\end{tabular}

$120 \times 120 \times 120$ with aggregates of size $10 \times 10 \times 10$

\begin{tabular}{c|ccccccc}
\hline 1 & 63 & 54 & 33 & 49 & 31 & 42 & 25 \\
2 & 23 & 17 & 12 & 16 & 11 & 13 & 9 \\
3 & 17 & 9 & 6 & 7 & 6 & 6 & 4 \\
4 & 10 & 7 & 4 & 5 & 4 & 4 & 3 \\
6 & 7 & 6 & 4 & 4 & 3 & 3 & 2 \\
8 & 7 & 6 & 3 & 3 & 3 & 2 & 2 \\
10 & 7 & 5 & 3 & 3 & 2 & 2 & 2 \\
12 & 6 & 5 & 3 & 3 & 2 & 2 & 2 \\
\hline \multicolumn{7}{c}{$120 \times 120 \times 120$} & with aggregates of size $20 \times 20 \times 20$ \\
\hline 1 & $100+$ & $100+$ & $100+$ & $100+$ & $100+$ & $100+$ & 93 \\
2 & 84 & 69 & 42 & 62 & 40 & 53 & 31 \\
3 & 43 & 33 & 21 & 30 & 21 & 25 & 16 \\
4 & 27 & 19 & 13 & 17 & 13 & 15 & 9 \\
6 & 17 & 8 & 6 & 8 & 6 & 6 & 4 \\
8 & 11 & 8 & 5 & 5 & 4 & 3 & 3 \\
10 & 8 & 6 & 4 & 4 & 3 & 3 & 2 \\
12 & 7 & 6 & 4 & 4 & 3 & 3 & 2
\end{tabular}


TABLE 8.2

Convergence rate by method and degree of the smoothing polynomial.

\begin{tabular}{|c|c|c|c|c|c|c|c|}
\hline$d$ & A6S5O & T5.1 & T5.1s & $\mathrm{T} 5.2 \mathrm{k} 2$ & T5.2sk2 & T5.2k3 & T5.2sk3 \\
\hline \multicolumn{8}{|c|}{$60 \times 60 \times 60$ with aggregates of size $10 \times 10 \times 10$} \\
\hline 1 & $8.11 \mathrm{e}-1$ & $8.08 \mathrm{e}-1$ & $6.58 \mathrm{e}-1$ & $7.88 \mathrm{e}-1$ & $6.29 \mathrm{e}-1$ & $7.64 \mathrm{e}-1$ & $5.90 \mathrm{e}-1$ \\
\hline 2 & $5.34 \mathrm{e}-1$ & $5.24 \mathrm{e}-1$ & $2.96 \mathrm{e}-1$ & $4.85 \mathrm{e}-1$ & $2.80 \mathrm{e}-1$ & $4.38 \mathrm{e}-1$ & $2.16 \mathrm{e}-1$ \\
\hline 3 & $3.60 \mathrm{e}-1$ & $2.24 \mathrm{e}-1$ & $9.27 \mathrm{e}-2$ & $2.08 \mathrm{e}-1$ & 8.37e-2 & $1.65 \mathrm{e}-1$ & $5.03 \mathrm{e}-2$ \\
\hline 4 & $2.01 \mathrm{e}-1$ & $1.53 \mathrm{e}-1$ & $2.43 \mathrm{e}-2$ & $6.43 e-2$ & $1.94 \mathrm{e}-2$ & $3.13 \mathrm{e}-2$ & $6.39 \mathrm{e}-3$ \\
\hline 6 & $1.19 \mathrm{e}-1$ & $1.46 \mathrm{e}-1$ & $1.45 \mathrm{e}-2$ & $3.06 \mathrm{e}-2$ & $2.90 \mathrm{e}-3$ & $1.51 \mathrm{e}-2$ & $1.42 \mathrm{e}-4$ \\
\hline 8 & $1.20 \mathrm{e}-1$ & $1.13 \mathrm{e}-1$ & $6.42 \mathrm{e}-3$ & $1.04 \mathrm{e}-2$ & $1.36 \mathrm{e}-3$ & $1.38 \mathrm{e}-3$ & $4.33 e-5$ \\
\hline 10 & $6.21 \mathrm{e}-2$ & $8.38 \mathrm{e}-2$ & $1.10 \mathrm{e}-2$ & $1.90 \mathrm{e}-2$ & $1.19 \mathrm{e}-4$ & $1.59 \mathrm{e}-3$ & $5.94 \mathrm{e}-6$ \\
\hline 12 & $5.65 \mathrm{e}-2$ & $8.81 \mathrm{e}-2$ & $8.06 \mathrm{e}-3$ & $1.07 \mathrm{e}-2$ & $2.30 \mathrm{e}-4$ & $1.23 \mathrm{e}-3$ & $4.15 \mathrm{e}-6$ \\
\hline \multicolumn{8}{|c|}{$60 \times 60 \times 60$ with aggregates of size $20 \times 20 \times 20$} \\
\hline 1 & $9.37 \mathrm{e}-1$ & $9.30 \mathrm{e}-1$ & $8.86 \mathrm{e}-1$ & $9.24 \mathrm{e}-1$ & $8.76 \mathrm{e}-1$ & $9.16 \mathrm{e}-1$ & $8.58 \mathrm{e}-1$ \\
\hline 2 & $8.43 \mathrm{e}-1$ & $8.41 \mathrm{e}-1$ & $7.10 \mathrm{e}-1$ & $8.21 \mathrm{e}-1$ & $6.90 \mathrm{e}-1$ & $8.02 \mathrm{e}-1$ & $6.39 \mathrm{e}-1$ \\
\hline 3 & $7.12 \mathrm{e}-1$ & $7.04 \mathrm{e}-1$ & $5.07 \mathrm{e}-1$ & $6.73 e-1$ & $4.89 \mathrm{e}-1$ & $6.38 \mathrm{e}-1$ & $4.05 \mathrm{e}-1$ \\
\hline 4 & $5.68 \mathrm{e}-1$ & $5.46 \mathrm{e}-1$ & $3.30 \mathrm{e}-1$ & $5.06 \mathrm{e}-1$ & $3.20 \mathrm{e}-1$ & $4.60 \mathrm{e}-1$ & $2.17 \mathrm{e}-1$ \\
\hline 6 & $3.67 \mathrm{e}-1$ & $2.25 \mathrm{e}-1$ & $8.97 \mathrm{e}-2$ & $2.05 \mathrm{e}-1$ & $8.33 \mathrm{e}-2$ & $1.64 \mathrm{e}-1$ & $3.16 \mathrm{e}-2$ \\
\hline 8 & $2.24 \mathrm{e}-1$ & $1.55 \mathrm{e}-1$ & $2.74 \mathrm{e}-2$ & $4.97 \mathrm{e}-2$ & $8.80 \mathrm{e}-3$ & $2.34 \mathrm{e}-2$ & $3.51 \mathrm{e}-3$ \\
\hline 10 & $1.70 \mathrm{e}-1$ & $1.53 \mathrm{e}-1$ & $2.34 \mathrm{e}-2$ & $4.40 \mathrm{e}-2$ & $7.10 \mathrm{e}-4$ & $1.36 \mathrm{e}-2$ & $2.49 \mathrm{e}-4$ \\
\hline 12 & $1.09 \mathrm{e}-1$ & $1.40 \mathrm{e}-1$ & $1.95 \mathrm{e}-2$ & $4.13 e-2$ & $1.59 \mathrm{e}-3$ & $1.53 \mathrm{e}-2$ & $5.67 e-5$ \\
\hline \multicolumn{8}{|c|}{$120 \times 120 \times 120$ with aggregates $\mathrm{f}$ size $10 \times 10 \times 10$} \\
\hline 1 & $7.89 \mathrm{e}-1$ & $8.02 \mathrm{e}-1$ & $6.29 \mathrm{e}-1$ & $7.81 \mathrm{e}-1$ & $6.11 \mathrm{e}-1$ & $7.57 \mathrm{e}-1$ & $5.52 \mathrm{e}-1$ \\
\hline 2 & $5.09 \mathrm{e}-1$ & $5.14 \mathrm{e}-1$ & $2.77 \mathrm{e}-1$ & $4.75 \mathrm{e}-1$ & $2.74 \mathrm{e}-1$ & $4.27 \mathrm{e}-1$ & $1.85 \mathrm{e}-1$ \\
\hline 3 & $3.64 \mathrm{e}-1$ & $1.77 \mathrm{e}-1$ & $7.37 \mathrm{e}-2$ & $1.77 \mathrm{e}-1$ & $6.86 \mathrm{e}-2$ & $1.55 \mathrm{e}-1$ & $4.13 \mathrm{e}-2$ \\
\hline 4 & $1.98 \mathrm{e}-1$ & $1.48 \mathrm{e}-1$ & $2.35 \mathrm{e}-2$ & $6.04 \mathrm{e}-2$ & $1.68 \mathrm{e}-2$ & $2.68 \mathrm{e}-2$ & $5.47 \mathrm{e}-3$ \\
\hline 6 & $1.03 \mathrm{e}-1$ & $1.34 \mathrm{e}-1$ & $1.25 \mathrm{e}-2$ & $2.09 \mathrm{e}-2$ & $3.44 \mathrm{e}-3$ & $1.40 \mathrm{e}-2$ & $1.33 \mathrm{e}-4$ \\
\hline 8 & $1.29 \mathrm{e}-1$ & $1.25 \mathrm{e}-1$ & $4.71 \mathrm{e}-3$ & $8.67 e-3$ & $1.58 \mathrm{e}-3$ & $1.31 \mathrm{e}-3$ & $4.36 \mathrm{e}-5$ \\
\hline 10 & $6.11 \mathrm{e}-2$ & $8.11 \mathrm{e}-2$ & $1.00 \mathrm{e}-2$ & $1.89 \mathrm{e}-2$ & $1.19 \mathrm{e}-4$ & $1.10 \mathrm{e}-3$ & $5.09 \mathrm{e}-6$ \\
\hline 12 & $6.24 \mathrm{e}-2$ & $9.10 \mathrm{e}-2$ & $1.05 \mathrm{e}-2$ & $1.35 \mathrm{e}-2$ & $4.30 \mathrm{e}-4$ & $2.02 \mathrm{e}-3$ & $7.58 \mathrm{e}-6$ \\
\hline \multicolumn{8}{|c|}{$120 \times 120 \times 120$ with aggregates of size $20 \times 20 \times 20$} \\
\hline 1 & $9.38 \mathrm{e}-1$ & $9.29 \mathrm{e}-1$ & $8.83 \mathrm{e}-1$ & $9.22 \mathrm{e}-1$ & $8.75 \mathrm{e}-1$ & $9.10 \mathrm{e}-1$ & $8.53 \mathrm{e}-1$ \\
\hline 2 & $8.38 \mathrm{e}-1$ & $8.40 \mathrm{e}-1$ & $7.03 \mathrm{e}-1$ & $8.17 \mathrm{e}-1$ & $6.88 \mathrm{e}-1$ & $8.04 \mathrm{e}-1$ & $6.26 \mathrm{e}-1$ \\
\hline 3 & $7.06 \mathrm{e}-1$ & $7.09 \mathrm{e}-1$ & $5.02 \mathrm{e}-1$ & $6.75 \mathrm{e}-1$ & $4.93 \mathrm{e}-1$ & $6.45 \mathrm{e}-1$ & $3.93 \mathrm{e}-1$ \\
\hline 4 & $5.62 \mathrm{e}-1$ & $5.55 \mathrm{e}-1$ & $3.33 \mathrm{e}-1$ & $5.12 \mathrm{e}-1$ & $3.31 \mathrm{e}-1$ & $4.73 \mathrm{e}-1$ & $2.06 \mathrm{e}-1$ \\
\hline 6 & $3.85 \mathrm{e}-1$ & $1.89 \mathrm{e}-1$ & $8.66 \mathrm{e}-2$ & $1.96 \mathrm{e}-1$ & $8.06 \mathrm{e}-2$ & $1.77 \mathrm{e}-1$ & $4.02 \mathrm{e}-2$ \\
\hline 8 & $2.27 \mathrm{e}-1$ & $1.55 \mathrm{e}-1$ & $2.62 \mathrm{e}-2$ & $5.67 \mathrm{e}-2$ & $1.89 \mathrm{e}-2$ & $2.66 \mathrm{e}-2$ & $5.78 \mathrm{e}-3$ \\
\hline 10 & $1.70 \mathrm{e}-1$ & $1.47 \mathrm{e}-1$ & $2.09 \mathrm{e}-2$ & $4.95 \mathrm{e}-2$ & $3.44 \mathrm{e}-3$ & $1.67 \mathrm{e}-2$ & $3.10 \mathrm{e}-4$ \\
\hline 12 & $1.18 \mathrm{e}-1$ & $1.44 \mathrm{e}-1$ & $1.44 \mathrm{e}-2$ & $3.33 \mathrm{e}-2$ & $2.35 \mathrm{e}-3$ & $1.44 \mathrm{e}-2$ & $9.82 \mathrm{e}-5$ \\
\hline
\end{tabular}

